J. DIFFERENTIAL GEOMETRY

84 (2010) 45-86

\title{
ON THE $\sigma_{2}$-SCALAR CURVATURE
}

\author{
Yuxin Ge, Chang-Shou Lin \& Guofang Wang
}

\begin{abstract}
In this paper, we establish an analytic foundation for a fully non-linear equation $\frac{\sigma_{2}}{\sigma_{1}}=f$ on manifolds with metrics of positive scalar curvature and apply it to give a (rough) classification of such manifolds. A crucial point is a simple observation that this equation is a degenerate elliptic equation without any condition on the sign of $f$ and it is elliptic not only for $f>0$ but also for $f<0$. By defining a Yamabe constant $Y_{2,1}$ with respect to this equation, we show that a manifold with metrics of positive scalar curvature admits a conformal metric of positive scalar curvature and positive $\sigma_{2}$-scalar curvature if and only if $Y_{2,1}>0$. We give a complete solution for the corresponding Yamabe problem. Namely, let $g_{0}$ be a positive scalar curvature metric, then in its conformal class there is a conformal metric with

$$
\sigma_{2}(g)=\kappa \sigma_{1}(g),
$$

for some constant $\kappa$. Using these analytic results, we give a rough classification of the space of manifolds with metrics of positive scalar curvature.
\end{abstract}

\section{Introduction}

Let $\left(M, g_{0}\right)$ be a compact Riemannian manifold of dimension $n$ with metric $g_{0}$ and $\left[g_{0}\right]$ the conformal class of $g_{0}$. Let $R i c_{g}$ and $R_{g}$ denote the Ricci tensor and scalar curvature of a metric $g$ respectively. The Schouten tensor of the metric $g$ is defined by

$$
S_{g}=\frac{1}{n-2}\left(\operatorname{Ric}_{g}-\frac{R_{g}}{2(n-1)} \cdot g\right) .
$$

The importance of the Schouten tensor in conformal geometry can be viewed in the following decomposition of the Riemann curvature tensor

$$
\text { Riem }_{g}=W_{g}+S_{g} \oplus g,
$$

where $\left(\mathbb{}\right.$ is the Kulkani-Nomizu product and $W_{g}$ is the Weyl tensor. Note that $g^{-1} \cdot W_{g}$ is invariant in a given conformal class. Therefore, in a conformal class the Schouten tensor is important.

Received 03/15/2007. 
The objective of this paper has two-folds. First, we study a class of fully nonlinear quotient equations relating to the scalar curvature and the recently introduced $\sigma_{2}$-scalar curvature. Then, we apply the analysis established for this class of equations to study the space of metrics of positive scalar curvature. Let us first recall the definition of the $\sigma_{2}$-scalar curvature.

For a given $1 \leq k \leq n$, the $\sigma_{k}$-scalar curvature or $k$-scalar curvature is defined by

$$
\sigma_{k}(g):=\sigma_{k}\left(g^{-1} \cdot S_{g}\right),
$$

where $g^{-1} \cdot S_{g}$ is locally defined by $\left(g^{-1} \cdot S_{g}\right)_{j}^{i}=\sum_{k} g^{i k}\left(S_{g}\right)_{k j}$ and $\sigma_{k}$ is the $k$ th elementary symmetric function. Here for an $n \times n$ symmetric matrix $A$ we define $\sigma_{k}(A)=\sigma_{k}(\Lambda)$, where $\Lambda=\left(\lambda_{1}, \cdots, \lambda_{n}\right)$ is the set of eigenvalues of $A$. It is clear that $\sigma_{1}(g)$ is a constant multiple of the scalar curvature $R_{g}$. The $\sigma_{k}$-scalar curvature $\sigma_{k}(g)$, which was first considered by Viaclovsky [60], is a natural generalization of the scalar curvature.

In this paper, we focus on the $\sigma_{2}$-scalar curvature. One of the reasons to restrict ourself to $\sigma_{2}$-scalar curvature is that $\sigma_{2}(g)$ still has a variational structure. This is an observation of Viaclovsky [60] (For $k>2$, $\sigma_{k}(g)$ has a variational structure if and only if the underlying manifold is locally conformally flat $[\mathbf{6 0}],[\mathbf{6}])$. The variational structure is very crucial for our paper.

Since [60] and [10], there has been an intensive study for a nonlinear Yamabe problem related to the $\sigma_{k}$-scalar curvature $\sigma_{k}(g)$, namely, finding a conformal metric $g$ in a given conformal class $\left[g_{0}\right]$ satisfying

$$
\sigma_{k}(g)=c,
$$

where

$$
g \in\left[g_{0}\right] \cap \Gamma_{k}^{+} .
$$

Here $\Gamma_{k}^{+}$is a convex open cone -the Garding cone- defined by

$$
\Gamma_{k}^{+}=\left\{\Lambda=\left(\lambda_{1}, \lambda_{2}, \cdots, \lambda_{n}\right) \in \mathbb{R}^{n} \mid \sigma_{j}(\Lambda)>0, \forall j \leq k\right\} .
$$

By $g \in \Gamma_{k}^{+}$we mean that the Schouten tensor $S_{g}(x) \in \Gamma_{k}^{+}$for any $x \in M$. $g \in \Gamma_{1}^{+}$is equivalent to that $g$ has positive scalar curvature. Note that the condition $g \in \Gamma_{k}^{+}$guarantees that equation (1) is elliptic.

Equation (1) is a fully nonlinear conformal equation, but it admits many nice properties, which usually are only true for the semilinear equations. For example, the local a priori estimates were established in $[\mathbf{2 6}]$, a Liouville type theorem was given in $[\mathbf{4 5}]$. The existence problem of (1) has been intensively studied. For the existence results see [11], $[18],[19],[22],[27],[29],[31],[33],[45],[46],[47][54],[59],[62]$. See also a recent survey by Viaclovsky $[\mathbf{6 3}]$ and references therein or the lecture notes of Guan [21]. There are many interesting applications in geometry, especially in the 4-dimensional case, see for examples [10], $[\mathbf{1 2}]$, and [64]. See also $[\mathbf{2 2}],[23]$. 
However, till now one can only deal with equations like

$$
\sigma_{k}(g)=f
$$

with condition $f \geq 0$ or even $f>0$. This has something to do with the ellipticity of the corresponding equation. With this restriction we could not use equation (3) to study negative $\sigma_{2}$-scalar curvature. (cf. the work of Gursky and Viaclovsky [34] for metrics in $\Gamma_{-}^{k}$ and a class of uniformly elliptic fully nonlinear equations [29].) In this paper, instead of $\sigma_{2}(g)$ we consider the quotient type equation

$$
\frac{\sigma_{2}}{\sigma_{1}}=f .
$$

When $f>0$, it was studied recently in $[\mathbf{1 9}]$. See also $[\mathbf{2 8}],[\mathbf{3 1}],[\mathbf{2 4}]$ and [54]. The crucial point of this paper is that we can also deal with the case with negative functions $f$ for (4). In fact, we have

Observation. The operator $\frac{\sigma_{2}}{\sigma_{1}}$ is elliptic in the cone $\Gamma_{1}^{+} \backslash \mathcal{R}_{1}$ and concave in $\Gamma_{1}^{+}$.

For the notation and the proof of this Observation, see Lemma 1. Recall that the ordinary Yamabe constant is defined

$$
Y_{1}\left(\left[g_{0}\right]\right)=Y_{1}\left(M,\left[g_{0}\right]\right):=\inf _{g \in \mathcal{C}_{1}\left(\left[g_{0}\right]\right)} \frac{\int \sigma_{1}(g) d \operatorname{vol}(g)}{(\operatorname{vol}(g))^{\frac{n-2}{n}}} .
$$

We will call it the first Yamabe constant. For simplicity of notation, we denote $\Gamma_{k}^{+} \cap\left[g_{0}\right]$ by $\mathcal{C}_{k}\left(\left[g_{0}\right]\right)$, or even just $\mathcal{C}_{k}$ if there is no confusion. A conformal class has positive first Yamabe constant if and only if this class contains a metric of positive scalar curvature. This is a simple, but important fact in the study of metrics of positive scalar curvature. As one of applications of our study of equation (4), we will prove a similar result for the $\sigma_{2}$-scalar curvature. From now on we consider the conformal class $[g]$ with $\mathcal{C}_{1} \neq \emptyset$ and call a metric of positive scalar curvature a psc metric. Now we first define a "nonlinear eigenvalue" for $\sigma_{2}(g)$, more precisely for a fully nonlinear operator

$$
\sigma_{2}\left(\nabla^{2} u+d u \otimes d u-\frac{|\nabla u|^{2}}{2} g_{0}+S_{g_{0}}\right),
$$

where $g=e^{-2 u} g_{0}$. Define

(6) $\lambda\left(g_{0}, \sigma_{2}\right)=\lambda\left(M, g_{0}, \sigma_{2}\right):= \begin{cases}\inf _{g \in \mathcal{C}_{1}\left(\left[g_{0}\right]\right)} \frac{\int \sigma_{2}(g) d v o l(g)}{\int e^{4 u} d v o l(g)}, & \text { if } n>4, \\ \int \sigma_{2}(g) d \operatorname{vol}(g), & \text { if } n=4, \\ \sup _{g \in \mathcal{C}_{1}\left(\left[g_{0}\right]\right)} \frac{\int \sigma_{2}(g) d v o l(g)}{\int e^{4 u} d v o l(g)}, & \text { if } n=3 .\end{cases}$ 
One can also define a similar constant $\lambda\left(g_{0}, \sigma_{k}\right)$ for $\sigma_{k}$. When $k=1$, one can check that $\lambda\left(g_{0}, \sigma_{k}\right)$ is the first eigenvalue of the conformal Laplacian. Hence we call the constant $\lambda\left(g_{0}, \sigma_{2}\right)$ nonlinear eigenvalue of operator (5). Such a nonlinear eigenvalue for fully nonlinear operator was first considered in [49]. See also [65]. In the context of fully nonlinear conformal operators, it was first considered in a preliminary version of $[\mathbf{2 7}]$.

Now we define the second conformal Yamabe constant for a conformal class with psc metrics.

$$
\begin{aligned}
& Y_{2,1}\left(\left[g_{0}\right]\right)=Y_{2,1}\left(M,\left[g_{0}\right]\right):= \\
& \begin{cases}\inf _{g \in \mathcal{C}_{1}\left(\left[g_{0}\right]\right)} \frac{\int \sigma_{2}(g) \operatorname{dvol}(g)}{\left(\int \sigma_{1}(g) \operatorname{dvol}(g)\right)^{\frac{n-4}{n-2}},} & \text { if } n>4, \\
\int \sigma_{2}(g) d \operatorname{vol}(g), & \text { if } n=4, \\
\sup _{g \in \mathcal{C}_{1}\left(\left[g_{0}\right]\right)} \int \sigma_{2}(g) \operatorname{dvol}(g) \times \int \sigma_{1}(g) \operatorname{dvol}(g), & \text { if } n=3 .\end{cases}
\end{aligned}
$$

We emphasize that the infimum (or supremum) in the definition of $\lambda_{2}$ and $Y_{2,1}$ is taken over $\mathcal{C}_{1}$, not over $\mathcal{C}_{2}$. It is well-known that when $n=4, \int_{M} \sigma_{2}(g) \operatorname{dvol}(g)$ is a constant in a given conformal class. The relationship between the sign of $\lambda\left(g_{0}, \sigma_{2}\right)$ and $Y_{2,1}\left(\left[g_{0}\right]\right)$ will be discussed in the next section.

Now we state our main analytic results in this paper .

Theorem 1. Assume $n \geq 3$. The constant $\lambda\left(g_{0}, \sigma_{2}\right)$ is achieved, provided $\lambda\left(g_{0}, \sigma_{2}\right) \geq 0$. More precisely, we have

1) If $\lambda\left(g_{0}, \sigma_{2}\right)>0$, then $\mathcal{C}_{2}\left(\left[g_{0}\right]\right)$ is not empty and $\lambda\left(g_{0}, \sigma_{2}\right)>0$ is achieved by a smooth metric $g=e^{-2 u} g_{0} \in \mathcal{C}_{2}$ satisfying

$$
\sigma_{2}(g)=\lambda e^{4 u} .
$$

2) If $\lambda\left(g_{0}, \sigma_{2}\right)=0$, then $\lambda\left(g_{0}, \sigma_{2}\right)=0$ is achieved by a $C^{1,1}$ metric $g=e^{-2 u} g_{0} \in \overline{\mathcal{C}}_{1}$ satisfying

$$
\sigma_{2}(g)=0 .
$$

Here $\overline{\mathcal{C}}_{1}$ is the closure of $\mathcal{C}_{1}$. Namely, $\overline{\mathcal{C}}_{1}$ is the space of metrics with non-negative scalar curvature. With the help of Theorem 1 and results in $[\mathbf{1 1}],[\mathbf{3 3}]$ and $[\mathbf{1 9}]$ we can solve the Yamabe problem for $\frac{\sigma_{2}}{\sigma_{1}}$ completely.

Theorem 2. Let $\left(M^{n}, g_{0}\right)$ be a compact Riemannian manifold with $g_{0} \in \Gamma_{1}^{+}$and $n \geq 3$. The following holds 
1) If $Y_{2,1}\left(\left[g_{0}\right]\right)>0$, then $\mathcal{C}_{2}\left(\left[g_{0}\right]\right)$ is not empty. Moreover there is a smooth metric $g$ in $\mathcal{C}_{2}$ satisfying

$$
\frac{\sigma_{2}(g)}{\sigma_{1}(g)}=1 \text {. }
$$

2) If $Y_{2,1}\left(\left[g_{0}\right]\right)=0$, then there is a $C^{1,1}$ metric $g$ in $\overline{\mathcal{C}}_{1}$ satisfying

$$
\sigma_{2}(g)=0 .
$$

3) If $Y_{2,1}\left(\left[g_{0}\right]\right)<0$, then there is a smooth metric $g$ in $\overline{\mathcal{C}}_{1}$ satisfying

$$
\sigma_{2}(g)=-\sigma_{1}(g) \text {. }
$$

In fact, we can show that $Y_{2,1}$ is achieved when $n>3$. This will be carried out elsewhere. We remark that in case 3) though the solution metric $g$ is in $\overline{\mathcal{C}}_{1}$, it is smooth. One can show that any point $x \in M$ with $\sigma_{2}(g)(x)=0$ (and hence $\sigma_{1}(g)(x)=0$ ) is Ricci-flat, i.e., Ric $(x)=0$. We believe that $g \in \mathcal{C}_{1}$.

A direct consequence of the main analytic results is

Corollary 1. Let $\left(M^{n}, g_{0}\right)$ be a closed Riemannian manifold of dimension $n>2$ with positive Yamabe constant $Y_{1}\left(\left[g_{0}\right]\right)>0$. If $Y_{2,1}\left(\left[g_{0}\right]\right)$ $>0$, then there is a metric $g \in\left[g_{0}\right]$ with $g \in \Gamma_{2}^{+}$, i.e., with

$$
R_{g}>0 \text { and } \sigma_{2}(g)>0 \text {. }
$$

Note that $Y_{2,1}\left(\left[g_{0}\right]\right)>0$ if and only if $\lambda\left(g_{0}, \sigma_{2}\right)>0$. See Lemma 3 below. When $n=4$, this result was proved by Chang-Gursky-Yang [10]. Namely, for a closed 4-dimensional manifold $M^{4}$, if there is a metric $g_{0}$ with $Y_{1}\left(\left[g_{0}\right]\right)>0$ and

$$
\int_{M^{4}} \sigma_{2}\left(g_{0}\right) d v o l\left(g_{0}\right)>0
$$

then there is a metric $g \in\left[g_{0}\right]$ with $R_{g}>0$ and $\sigma_{2}(g)>0$. Another direct proof was given in $[\mathbf{3 2}]$. See also $[\mathbf{2 2}]$ for locally conformally flat manifolds. Many interesting applications to geometry were given in these papers, especially in [12]. This result of Chang-Gursky-Yang is in fact one of our main motivations of this paper. From their result, it is natural to ask if this is also true for 3 dimensional manifolds. Corollary 1 gives an affirmative answer. For convenience of the reader, we restate Corollary 1 for $n=3$.

Corollary 2. Let $\left(M^{3}, g_{0}\right)$ be a closed manifold of dimension 3 with positive Yamabe constant $Y_{1}\left(\left[g_{0}\right]\right)>0$ and $g_{0} \in \Gamma_{1}^{+}$such that

$$
\int_{M^{3}} \sigma_{2}\left(g_{0}\right) \operatorname{dvol}\left(g_{0}\right)>0
$$

then there is a metric $g \in\left[g_{0}\right]$ with $g \in \Gamma_{2}^{+}$, i.e., with

$$
R_{g}>0 \text { and } \sigma_{2}(g)>0 \text {. }
$$


Together with the Hamilton's result [35] obtained by the Ricci flow, the Corollary gives

Corollary 3. Let $\left(M^{3}, g_{0}\right)$ be a compact 3-dimensional manifold. If $Y_{1}\left(\left[g_{0}\right]\right)>0$ and $Y_{2,1}\left(\left[g_{0}\right]\right)>0$, then $M$ is diffeomorphic to a quotient of the sphere.

The difference between $n=4$ and $n=3$ is follows: In $n=4$, due to the conformal invariance of $\int_{M^{4}} \sigma_{2}(g) d v o l(g)$, Condition (11) implies that $\int_{M^{4}} \sigma_{2}(g) d v o l(g)>0$ for all $g \in\left[g_{0}\right]$. This is not the case for $n=3$. In fact one can show that for any 3 -dimensional manifold $\left(M^{3}, g_{0}\right)$ there is always a conformal metric $g \in\left[g_{0}\right]$ with $\int_{M^{3}} \sigma_{2}(g) d v o l(g)<0$ by using a method given in [20].

We remark that the method given in $[\mathbf{3 2}]$ and $[\mathbf{2 2}]$ can be used to give another proof of Corollary 1 at least for $n>4$. In another direction, one may ask if the non-emptyness of $\Gamma_{2}^{+}$implies the positivity of $Y_{2,1}$. This question is not easy to answer by only using the methods given in $[\mathbf{3 2}]$ and $[\mathbf{2 2}]$. In [19], we showed that the non-emptyness of $\Gamma_{2}^{+}$implies the positivity of

$$
\inf _{g \in \mathcal{C}_{2}}\left(\frac{1}{\left.\int \sigma_{1}(g) d v o l(g)\right)}\right)^{\frac{n-4}{n-2}} \int \sigma_{2}(g) d v o l(g) .
$$

It is clear that $Y_{2,1}$ is less than or equals to the above constant. With the analysis established here we can show that the non-emptyness of $\Gamma_{2}^{+}$ implies the positivity of $Y_{2,1}$.

Theorem 3. Let $(M, g)$ be a compact Riemannian manifold with positive scalar curvature. The second Yamabe constant is positive if and only if $\Gamma_{2}^{+}$is non-empty.

We remark again that the case $n=4$ was given in [10]. As mentioned above, it is well-known that the positivity of the first Yamabe constant $Y_{1}$ is equivalent to the existence of a conformal metric of positive scalar curvature. Theorem 3 means that $Y_{2,1}$ has a similar property. Motivated by this result, we hope to use $\sigma_{2}$-scalar curvature to give a further classification of the manifolds admitting metric with positive scalar curvature. This is the second aim of this paper. For the further discussion let us first recall the following definition.

$\left(1_{+}\right)$Closed connected manifolds with a Riemannian metric whose scalar curvature is non-negative and not identically 0 .

$\left(1_{0}\right)$ Closed connected manifolds with a Riemannian metric with nonnegative scalar curvature, but not in class $\left(1_{+}\right)$.

$\left(1_{-}\right)$Closed connected manifolds not in classes $\left(1_{+}\right)$or $\left(1_{0}\right)$.

There is a remarkable result of Kazdan and Warner obtained in 1975. 
Theorem A (Trichotomy Theorem) $([\mathbf{4 1}],[\mathbf{4 2}])$ Let $M^{n}$ be a closed connected manifold of dimension $n \geq 3$.

1. If $M$ belongs to class $\left(1_{+}\right)$, then every smooth function is the scalar curvature function for some Riemannian metric on $M$.

2. If $M$ belongs to class $\left(1_{0}\right)$, then a smooth function $f$ is the scalar curvature function of some Riemannian metric on $M$ if and only if $f(x)<0$ for some point $x \in M$, or else $f=0$. If the scalar curvature of some $g$ vanishes identically, then $g$ is Ricci flat.

3. If $M$ belongs to class (1-), then a smooth function $f$ is the scalar curvature function of some Riemannian metric on $M$ if and only if $f(x)<0$ for some point $x \in M$.

The analysis used in the proof of Theorem A is based on the analysis for the eigenvalue problem for the conformal Laplacian operator

$$
-\Delta v+\frac{n-2}{4(n-1)} R_{g} v=\lambda v
$$

where $\Delta$ is the Laplacian with respect to $g$ and $v$ is related to $u$ by $v=e^{-\frac{n-2}{2} u}$. And it is closely related to the famous Yamabe equation

$$
-\Delta v+\frac{n-2}{4(n-1)} R_{g} v=k v^{\frac{n+2}{n-2}}
$$

where $k$ is a constant. The existence of solutions for (13) is the so-called Yamabe problem, which was solved by Yamabe, Trudinger, Aubin and Scheon completely.

From Theorem A or an earlier result of Aubin [2] we know that a negative function can always be realized as a scalar function of a metric. See also the results of $[\mathbf{1 7}]$ and $[\mathbf{5 0}]$ for the existence of negative Ricci curvature. The class of $\left(1_{0}\right)$ is very small and consisting of very special manifolds, thanks to a result of Futaki [15]. Theorem A also implies that class $\left(1_{+}\right)$is just the class of manifolds which admit a metric of positive scalar curvature. There are topological obstructions for the manifolds of positive scalar curvature, see $[\mathbf{4 8}]$ and $[\mathbf{3 8}]$. This class attracts much attention of geometers for many years, especially after the work of Gromov-Lawson[20] and Schoen-Yau [53]. The most important problem in this field is the Gromov-Lawson-Rosenberg conjecture which was proved by Stolz [55] in the simply connected case. For this conjecture, see for instance [51] and [57].

Note that it is well-known that class $\left(1_{+}\right)$is equivalent to

$\left(1_{+}^{\prime}\right)$ Closed connected manifolds with a Riemannian metric whose scalar curvature is positive.

Now using $\sigma_{2}$-scalar curvature we divide $\left(1_{+}\right)$further into 3 subclasses: 
$\left(2_{+}\right)$Closed connected manifolds admitting a psc metric whose $\sigma_{2^{-}}$ scalar curvature is positive.

$\left(2_{0}\right)$ Closed connected manifolds admitting a psc metric with nonnegative $\sigma_{2}$-scalar curvature, but not in class $\left(2_{+}\right)$.

$\left(2_{-}\right)$Closed connected manifolds in $\left(1_{+}\right)$, but not in classes $\left(2_{+}\right)$or $\left(2_{0}\right)$.

Remark 1. We believe that class $\left(2_{+}\right)$is equivalent to the class of closed connected manifolds admitting a psc metric whose $\sigma_{2}$-scalar curvature is non-negative and not identically 0 . At the moment we could not prove this equivalence.

Analog to the Trichotomy Theorem of Kazdan-Warner for the scalar curvature and in view of the analysis established here, we propose the following

Conjecture (Trichotomy Theorem) Let $M^{n}(n>2)$ be a closed connected manifold in class $\left(1_{+}\right)$.

1. If $M$ belongs to class $\left(2_{+}\right)$, then for every smooth function $f$ there is a psc metric $g$ such that $f \sigma_{1}(g)$ is its $\sigma_{2}$-scalar curvature.

2. If $M$ belongs to class $\left(2_{0}\right)$, then for a smooth function $f, M$ admits a psc metric $g$ with $\sigma_{2}(g)=f \sigma_{1}(g)$ if and only if $f(x)<0$ for some point $x \in M$, or else $f=0$.

3 . If $M$ belongs to class $\left(2_{-}\right)$, then for a smooth function $f, M$ admits a psc metric $g$ with $\sigma_{2}(g)=f \sigma_{1}(g)$ if and only if $f(x)<0$ for some point $x \in M$.

Though we could not prove this conjecture at moment, we have the following results support this conjecture.

Theorem 4. Any Riemannian manifold $M^{n}(n \geq 4)$ in the class $\left(1_{+}\right)$ admits a metric with non-positive $\sigma_{2}$-scalar curvature and non-negative scalar curvature.

The metric given in Theorem 4 satisfies $\int \sigma_{2}(g) d v o l(g)<0$ and has vanishing Ricci curvature at points with $\sigma_{1}(g)(x)=0$.

Theorem 5. Let $M^{n}$ be a closed connected manifold of dimension $n>3$.

1. If $M$ belongs to class $\left(2_{+}\right)$, then for every constant $b$ there is a metric $g$ (with non-negative scalar curvature when $b \leq 0$ and positive scalar curvature when $b>0)$ such that $b \sigma_{1}(g)$ is its $\sigma_{2}$-scalar curvature.

2. If $M$ belongs to class $\left(2_{0}\right)$, then for a constant $b, M$ admits a metric $g$ (with non-negative scalar curvature when $b \leq 0$ and positive scalar curvature when $b>0)$ such that $\sigma_{2}(g)=b \sigma_{1}(g)$ if and only if $b \leq 0$. 
3. If $M$ belongs to class $\left(2_{-}\right)$, then for a constant $b, M$ admits a metric $g$ (with non-negative scalar curvature when $b \leq 0$ and positive scalar curvature when $b>0)$ such that $\sigma_{2}(g)=b \sigma_{1}(g)$ if and only if $b<0$.

The metrics found in Theorem 5 may have points with vanishing $\sigma_{1^{-}}$ scalar curvature. At such points its Ricci tensor also vanishes. When $b>0$, then the metric has positive scalar curvature. It is trivial that the sphere belongs to $\left(2_{+}\right)$. By Corollary 3, in 3-dimension, only the sphere and its quotients belong to $\left(2_{+}\right)$and there are no manifolds in $\left(2_{0}\right)$. In 4-dimension, many examples of manifolds with metrics in $\Gamma_{2}^{+}$ can be found in [10]. Those manifolds certainly belong to $\left(2_{+}\right)$. One can prove that $\mathbb{S}^{3} \times \mathbb{S}^{1}$ belongs to $\left(2_{0}\right)$, namely there is no positive scalar metric on $\mathbb{S}^{3} \times \mathbb{S}^{1}$ with positive $\sigma_{2}$-scalar curvature, see Section 7 below. Like class $\left(1_{0}\right)$, class $\left(2_{0}\right)$ should be very small. However, when $n>4$, we have no example of manifolds in class $\left(1_{+}\right)$which do not belong to $\left(2_{+}\right)$. This is somewhat strange. A possible candidate is the manifold $\mathbb{S}^{6} \times H^{3}$, where $H^{3}$ is a compact quotient of the hyperbolic space $\mathbb{H}^{3}$. It is easy to check that its product metric $g_{P}$ satisfies that $\sigma_{1}\left(g_{P}\right)>0$ and $\sigma_{2}\left(g_{P}\right)=0$. However, we believe that $\mathbb{S}^{6} \times \mathbb{H}^{3}$ has a metric $g$ with $\sigma_{1}(g)>0$ and $\sigma_{2}(g)>0$. See Example 2 in Section 7. Therefore we may ask

Problem. Is there a topological obstruction for the existence of psc metric with positive $\sigma_{2}$-scalar curvature when $n>4$ ?

For the scalar curvature as mentioned above there are topological obstructions. What we ask is to find further conditions to distinguish manifolds between $\left(2_{+}\right),\left(2_{0}\right)$ and $\left(2_{+}\right)$for higher dimensional manifolds. There is a similar and related problem proposed by Stolz in [56] for further obstructions to the existence of metrics with positive Ricci tensor. For some relationship between the positive Ricci tensor and positive $\sigma_{k}$-scalar curvature, see [25]. With our analysis, the problem to find a topological obstruction for $\sigma_{2}$-scalar curvature perhaps might be not very difficult.

The paper is organized as follows. In Section 2, we show the Observation and discuss the relationship between $\lambda\left(g_{0}, \sigma_{2}\right)$ and $Y_{2,1}$. We introduce a class of perturbed equations (28) and a Yamabe type flow (31) in Section 3 and establish local a priori estimates for these equations and flows in Section 4. In Section 5 we prove the global existence of the Yamabe type flow and Theorem 1 and Theorem 2. One of another crucial points of this paper, the Yamabe type flow preserves the positivity of the scalar curvature, will also be proved in this section. In Section 6 , we show Theorem 3 . We give the geometric applications in 
Section 7 by proving Theorems 4 and 5 . In the last section, we mention further applications in similar equations.

Acknowledgments. This project was started several years ago together with Pengfei Guan. The key observation was discovered by him and the second named author in their study of fully nonlinear equations. We would like to thank him for many helpful discussions and encouragement. A part of the work was carried out while the first author was visiting McGill University and he would like to thank the department and Pengfei Guan for warm hospitality.

After the paper has been circulated and submitted, the first and third authors were kindly informed by Paul Yang in a conference in CIRM in June 2007 that Catino and Djadli just announced a similar result to Corollary 2. The preprint appeared later in Arxiv [9].

We would like to thank the referees for their critical reading and useful suggestions.

\section{Some preliminary facts}

Let $\mathcal{S}_{n}$ be the space of $n \times n$ real symmetric matrices and $F$ a smooth function in $\mathcal{S}_{n}$. By extending $F$ to the whole space of $n \times n$ real matrices by $F(A)=F\left(\frac{1}{2}\left(A+A^{t}\right)\right)$, we view $F$ as a function of $n \times n$ variables $w_{i j}$ and define

$$
F^{i j}=\frac{\partial F}{\partial w_{i j}}
$$

The quotient function $\frac{\sigma_{2}}{\sigma_{1}}$ can be viewed as a function in $\mathcal{S}_{n}$ as follows. Let $W$ be a symmetric matrix and $\Lambda_{W}=\left\{\lambda_{1}, \lambda_{2}, \cdots, \lambda_{n}\right\}$ its eigenvalues. Then we define $\frac{\sigma_{2}}{\sigma_{1}}(W)=\frac{\sigma_{2}\left(\Lambda_{W}\right)}{\sigma_{1}\left(\Lambda_{W}\right)}$. A symmetric matrix is said to be in $\Gamma_{k}^{+}$if $\Lambda_{W} \in \Gamma_{k}^{+}$. Let $\mathcal{R}_{1}$ be the subspace of $\mathcal{S}_{n}$ consisting of symmetric matrices of rank 1 .

Lemma 1. For $1<k \leq n$ set $F=\frac{\sigma_{k}}{\sigma_{k-1}}$. We have

1) the matrix $\left(F^{i j}\right)(W)$ is semi-positive definite at $W \in \Gamma_{k-1}^{+}$and is positive definite at $W \in \Gamma_{k-1}^{+} \backslash \mathcal{R}_{1}$.

2) The function $F$ is concave in the cone $\Gamma_{k-1}^{+}$. When $k=2$, for all

$$
W \in \Gamma_{1}^{+} \text {and for all } R=\left(r_{i j}\right) \in \mathcal{S}_{n} \text {, we have }
$$

$$
\sum_{i j k l} \frac{\partial^{2}}{\partial w_{i j} \partial w_{k l}}\left(\frac{\sigma_{2}(W)}{\sigma_{1}(W)}\right) r_{i j} r_{k l}=-\frac{\sum_{i j}\left(\sigma_{1}(W) r_{i j}-\sigma_{1}(R) w_{i j}\right)^{2}}{\sigma_{1}^{3}(W)}
$$


Proof. For $\Lambda=\left(\lambda_{1}, \lambda_{2}, \cdots, \lambda_{n}\right)$ and $i \in\{1,2, \cdots, n\}$ let $\Lambda_{i}=\{1,2, \cdots$, $\hat{i}, \cdots, n\}$ be the $(n-1)$-tuple obtained from $\Lambda$ without the $i$ th-component. A direct calculation gives

$$
\frac{\partial F}{\partial \lambda_{i}}=\frac{\sigma_{k-1}\left(\Lambda_{i}\right) \sigma_{k-1}(\Lambda)-\sigma_{k}(\Lambda) \sigma_{k-2}\left(\Lambda_{i}\right)}{\sigma_{k-1}(\Lambda)^{2}} .
$$

Since $\Lambda \in \Gamma_{k-1}^{+}, \Lambda_{i} \in \Gamma_{k-2}^{+}$. For the proof see for instance [39]. From two identities

$$
\sigma_{k}(\Lambda)=\lambda_{i} \sigma_{k-1}\left(\Lambda_{i}\right)+\sigma_{k}\left(\Lambda_{i}\right) \text { and } \sigma_{k-1}(\Lambda)=\lambda_{i} \sigma_{k-2}\left(\Lambda_{i}\right)+\sigma_{k-1}\left(\Lambda_{i}\right),
$$

(15) becomes

$$
\frac{\partial F}{\partial \lambda_{i}}=\frac{\sigma_{k-1}^{2}\left(\Lambda_{i}\right)-\sigma_{k}\left(\Lambda_{i}\right) \sigma_{k-2}\left(\Lambda_{i}\right)}{\sigma_{k-1}(\Lambda)^{2}}
$$

Note that for convenience we set $\sigma_{0}(\Lambda)=1$. By the Newton-McLaughlin inequality

$$
(k-1)(n-k) \sigma_{k-1}^{2}\left(\Lambda_{i}\right) \geq k(n-k+1) \sigma_{k}\left(\Lambda_{i}\right) \sigma_{k-2}\left(\Lambda_{i}\right),
$$

we have

$$
\frac{\partial F}{\partial \lambda_{i}} \geq 0
$$

and equality implies that $\Lambda_{i}=\{0,0, \cdots, 0\}$, i.e., $\Lambda=\left\{0, \cdots, 0, \lambda_{i}\right.$, $0, \cdots, 0\}$. This proves 1 ).

2) was proved in $[39]$.

q.e.d.

Though Lemma 1 is a rather simple fact, as mentioned in the introduction it is one of crucial points of our paper. (We remark that this Lemma might be observed also by other mathematicians. For example in $[\mathbf{1 4}]$ there is a rather similar formula. We would like to thank Tobias Lamm who told us this reference.) It means that $\frac{\sigma_{2}}{\sigma_{1}}$ is a concave, degenerate elliptic operator in $\Gamma_{1}^{+}$. With this observation in mind, we consider a family of perturbed operators for a positive number $\nu \in \mathbb{R}^{+}$

$$
\begin{aligned}
F_{\nu}: \Gamma_{1}^{+} & \rightarrow \mathbb{R} \\
W & \mapsto F_{\nu}(W)=\frac{\sigma_{2}(W)-\nu}{\sigma_{1}(W)} .
\end{aligned}
$$

As a direct consequence of Lemma 1, we have the following:

Lemma 2. The matrix $\left(F_{\nu}^{i j}\right)(W)$ is positive definite for all $W \in \Gamma_{1}^{+}$ and the function $F_{\nu}$ is strictly concave in $\Gamma_{1}^{+}$. Moreover, for all $W \in \Gamma_{1}^{+}$ and for all $R=\left(r_{i j}\right) \in \mathcal{S}_{n}(\mathbb{R})$

$$
\sum_{i j k l} \frac{\partial^{2} F_{\nu}(W)}{\partial w_{i j} \partial w_{k l}} r_{i j} r_{k l} \leq-\frac{2 \nu}{\sigma_{1}^{3}(W)}\left(\sum_{i} r_{i i}\right)^{2} .
$$


Lemma 2 means that for any positive number $\nu>0$, the operator $F_{\nu}$ is elliptic and strictly concave.

Now we discuss the relationship between the sign of the invariants $Y_{2,1}\left(\left[g_{0}\right]\right)$ and that of $\lambda\left(g_{0}, \sigma_{2}\right)$ defined in the introduction.

Lemma 3. The eigenvalue $\lambda\left(g_{0}, \sigma_{2}\right)$ is a finite number. Moreover, we have

(1) if $n \geq 4$, then $\lambda\left(g_{0}, \sigma_{2}\right)>0$ (resp. $=0,<0$ ) if and only if $Y_{2,1}\left(\left[g_{0}\right]\right)>0($ resp. $=0,<0)$;

(2) if $n=3$, then $\lambda\left(g_{0}, \sigma_{2}\right)>0$ (resp. $\left.\leq 0\right)$ if and only if $Y_{2,1}\left(\left[g_{0}\right]\right)>0$ $($ resp. $\leq 0)$.

Proof. The following formula was given in the proof of the Sobolev inequality in [18]: for any $g=e^{-2 u} g_{0} \in\left[g_{0}\right]$ we have

$$
\begin{aligned}
& 2 \int \sigma_{2}(g) d \operatorname{vol}(g) \\
= & \frac{n-4}{2} \int \sigma_{1}(g)|\nabla u|_{g_{0}}^{2} e^{2 u} d \operatorname{vol}(g)+\frac{n-4}{4} \int|\nabla u|_{g_{0}}^{4} e^{4 u} d v o l(g) \\
& +\int \sum_{i, j} T^{i j} S\left(g_{0}\right)_{i j} d v o l(g)+\int \sum_{i, j} S\left(g_{0}\right)^{i j} u_{i} u_{j} d v o l(g) \\
& +\frac{1}{2} \int \sigma_{1}\left(g_{0}\right)|\nabla u|_{g_{0}}^{2} e^{4 u} d \operatorname{vol}(g),
\end{aligned}
$$

where $T^{i j}=\sigma_{1}(W) g^{i j}-W^{i j}$ is the first Newton transformation associated with $W$. From this formula, we have

$$
\begin{aligned}
& 2 \int \sigma_{2}(g) d v o l(g) \\
= & \frac{n-4}{2} \int \sigma_{1}(g)|\nabla u|_{g_{0}}^{2} e^{2 u} d v o l(g)+\frac{n-4}{4} \int|\nabla u|_{g_{0}}^{4} e^{4 u} d v o l(g) \\
& +\int e^{2 u} \sigma_{1}(g) \sigma_{1}\left(g_{0}\right) d v o l(g)-\int e^{4 u}\left|S\left(g_{0}\right)\right|_{g_{0}}^{2} d v o l(g) \\
& +(4-n) \int \sum_{i, j} S\left(g_{0}\right)^{i j} u_{i} u_{j} d \operatorname{vol}(g)+\int \sigma_{1}\left(g_{0}\right)|\nabla u|_{g_{0}}^{2} e^{4 u} d \operatorname{vol}(g) \\
& +\int e^{4 u}\left\langle\nabla u, \nabla \sigma_{1}\left(g_{0}\right)\right\rangle_{g_{0}} d \operatorname{dvol}(g) .
\end{aligned}
$$


We first consider the case $n \geq 5$. By (20), there exists some $c>0$ such that

(21)

$$
\int \sigma_{2}(g) \operatorname{dvol}(g) \geq \frac{n-4}{16} \int|\nabla u|_{g_{0}}^{4} e^{4 u} d v o l(g)-c \int e^{4 u} d v o l(g),
$$

provided that $g \in \Gamma_{1}^{+}$. Here we have used the fact

$$
\begin{aligned}
& \left|\int \sum_{i, j} S\left(g_{0}\right)^{i j} u_{i} u_{j} d v o l(g)\right| \\
\leq & \frac{1}{8} \int|\nabla u|_{g_{0}}^{4} e^{4 u} d v o l(g)+2 \sup _{M}\left|S\left(g_{0}\right)\right|_{g_{0}}^{2} \int e^{4 u} d v o l(g)
\end{aligned}
$$

and

$$
\left|\int e^{4 u}\left\langle\nabla u, \nabla \sigma_{1}\left(g_{0}\right)\right\rangle_{g_{0}}\right| \leq \int \sigma_{1}\left(g_{0}\right)|\nabla u|_{g_{0}}^{2} e^{4 u} d \operatorname{vol}(g)+c \int e^{4 u} d \operatorname{vol}(g) .
$$

As a consequence, we conclude

$$
\lambda\left(g_{0}, \sigma_{2}\right) \geq-c
$$

Similarly, in case $n=3$, we have for all $g \in \mathcal{C}_{1}\left(\left[g_{0}\right]\right)$

$$
\begin{aligned}
& \int \sigma_{2}(g) d v o l(g) \\
= & -\frac{1}{4} \int \sigma_{1}(g)|\nabla u|_{g_{0}}^{2} e^{2 u} d v o l g-\frac{1}{8} \int|\nabla u|_{g_{0}}^{4} e^{4 u} d v o l(g) \\
& -\frac{1}{4} \int e^{4 u}|\nabla u|_{g_{0}}^{2} \sigma_{1}\left(g_{0}\right) d v o l(g) \\
& +\frac{1}{2} \int e^{4 u}\left(\sigma_{1}^{2}\left(g_{0}\right)-\left|S\left(g_{0}\right)\right|_{g_{0}}^{2}\right) \operatorname{dvol}(g) \\
& +\frac{1}{2} \int \sum_{i, j} S\left(g_{0}\right)^{i j} u_{i} u_{j} d v o l(g) \\
\leq & -\frac{1}{16} \int|\nabla u|_{g_{0}}^{4} e^{4 u} d \operatorname{vol}(g)+c \int e^{4 u} \operatorname{dvol}(g),
\end{aligned}
$$

which yields $\lambda\left(g_{0}, \sigma_{2}\right) \leq c$. Thus, we finish the proof of the first part of Lemma.

Now we begin to prove the second part. It is easy to see that for all $g \in \mathcal{C}_{1}\left(\left[g_{0}\right]\right)$ 


$$
\begin{aligned}
& \int_{M} \sigma_{1}(g) d v o l(g) \\
= & \int\left(\frac{n-2}{2}|\nabla u|^{2}+\sigma_{1}\left(g_{0}\right)\right) e^{2 u} d v o l(g) \geq Y_{1}\left(\left[g_{0}\right]\right)(\operatorname{Vol}(g))^{\frac{n-2}{n}} .
\end{aligned}
$$

Now we consider the case $n \geq 5$. Suppose $\lambda\left(g_{0}, \sigma_{2}\right)>0$, i.e.,

$$
\int_{M} \sigma_{2}(g) \operatorname{dvol}(g) \geq \lambda\left(g_{0}, \sigma_{2}\right) \int_{M} e^{4 u} d \operatorname{vol}(g)
$$

for any $g=e^{-2 u} g_{0} \in \Gamma_{1}^{+}$. From (21), (23) and Hölder's inequality, we deduce

$$
\begin{aligned}
\int \sigma_{2}(g) \operatorname{dvol}(g) & \geq c\left(\int|\nabla u|_{g_{0}}^{4} e^{4 u} d \operatorname{vol}(g)+\int e^{4 u} d \operatorname{vol}(g)\right) \\
& \geq c\left(\int_{M} \sigma_{1}(g) \operatorname{dvol}(g)\right)^{2}(\operatorname{Vol}(g))^{-1} \\
& \geq c Y_{1}\left(\left[g_{0}\right]\right)^{\frac{n}{n-2}}\left(\int_{M} \sigma_{1}(g) \operatorname{dvol}(g)\right)^{\frac{n-4}{n-2}}
\end{aligned}
$$

which implies $Y_{2,1}\left(\left[g_{0}\right]\right)>0$. Conversely, using Hölder's inequality and (23), we have

$$
\begin{aligned}
\int \sigma_{2}(g) \operatorname{dvol}(g) & \geq c\left(\int_{M} \sigma_{1}(g) \operatorname{dvol}(g)\right)^{\frac{n-4}{n-2}} \\
& \geq c Y_{1}\left(\left[g_{0}\right]\right)^{\frac{n-4}{n-2}}(\operatorname{Vol}(g))^{\frac{n-4}{n}} \geq c \int e^{4 u} \operatorname{dvol}(g) .
\end{aligned}
$$

This gives the desired result. Clearly, we have $\lambda\left(g_{0}, \sigma_{2}\right)<0$ if and only if $Y_{2,1}\left(\left[g_{0}\right]\right)<0$. Consequently, $\lambda\left(g_{0}, \sigma_{2}\right)=0$ if and only if $Y_{2,1}\left(\left[g_{0}\right]\right)=0$. In the cases $n=4$ and $n=3$, the result is trivial.

q.e.d.

Remark 2. In the case $n=3$, if $\lambda\left(g_{0}, \sigma_{2}\right)=\lambda<0$, then $Y_{2,1}\left(\left[g_{0}\right]\right)<$ 0 . To see this, for any $g \in \mathcal{C}_{1}\left(\left[g_{0}\right]\right)$, it follows from Hölder's inequality and (23) that there holds

$$
\begin{aligned}
& \int \sigma_{1}(g) d \operatorname{vol}(g) \int \sigma_{2}(g) d v o l(g) \\
\leq & \lambda \int \sigma_{1}(g) \operatorname{dvol}(g) \int e^{4 u} d \operatorname{vol}(g) \\
\leq & c \lambda \int e^{-u} d \operatorname{vol}\left(g_{0}\right) \int e^{u} d \operatorname{vol}\left(g_{0}\right) \leq c \lambda<0 .
\end{aligned}
$$


Remark 3. In the case $n \geq 5$, the invariant $Y_{2,1}\left(\left[g_{0}\right]\right)$ is finite real number. To see this, for any $g \in \mathcal{C}_{1}\left(\left[g_{0}\right]\right)$ it follows from the Hölder's inequality, (21) and (23) that

$$
\begin{aligned}
\frac{\int \sigma_{2}(g) d \operatorname{vol}(g)}{\left(\int \sigma_{1}(g) \operatorname{dvol}(g)\right)^{\frac{n-4}{n-2}}} & \geq-c \frac{\int e^{4 u} d \operatorname{vol}(g)}{\left(\int \sigma_{1}(g) d v o l(g)\right)^{\frac{n-4}{n-2}}} \\
& \geq-c \frac{(\operatorname{Vol}(g))^{\frac{n-4}{n}}}{\left(\int \sigma_{1}(g) d v o l(g)\right)^{\frac{n-4}{n-2}}} \geq-c>-\infty .
\end{aligned}
$$

In the case $n=3$, we do not know if $Y_{2,1}\left(\left[g_{0}\right]\right)<+\infty$ or not, although it is always true that $Y_{2,1}\left(\left[g_{0}\right]\right)>0$ if and only if $\lambda\left(g_{0}, \sigma_{2}\right)>0$, and that $\lambda\left(g_{0}, \sigma_{2}\right)$ is finite. However, we believe that it is true.

\section{Yamabe type flows}

Now we want to consider the existence of the following equation

$$
F_{\nu}(g)=\frac{\sigma_{2}(g)-\nu e^{4 u}}{\sigma_{1}}=\text { constant }
$$

with $g=e^{-2 u} g_{0}$ and $\nu>0$ a positive number (we could consider $\nu$ : $M \rightarrow \mathbb{R}^{+}$a positive function, but in this paper we will choose $\nu$ as a small positive constant). Following $[\mathbf{2 7}],[\mathbf{1 8}]$ and $[\mathbf{1 9}]$ we will introduce a suitable Yamabe type flow to study equation (28).

For any $\nu \in(0,+\infty)$ and for $g=e^{-2 u} g_{0}$, consider the following perturbed functional

$$
\mathcal{E}_{\nu}(g):= \begin{cases}\frac{2}{n-4} \int_{M}\left(\sigma_{2}(g)-\nu e^{4 u}\right) \operatorname{dvol}(g), & \text { if } n \neq 4, \\ -\int_{0}^{1} \int_{M}\left(\sigma_{2}\left(g_{t}\right)-2 \nu e^{4 t u}\right) u d v o l\left(g_{t}\right) d t, & \text { if } n=4,\end{cases}
$$

where $g_{t}=e^{-2 t u} g_{0}$. When $\nu=0$, the functional was considered in [60], $[\mathbf{1 0}]$ and $[\mathbf{7}]$. Set

$$
\mathcal{F}_{1}(g)=\int_{M} \sigma_{1}(g) \operatorname{dvol}(g) \quad \text { and } \quad \mathcal{F}_{2}(g)=\int_{M} \sigma_{2}(g) d \operatorname{vol}(g)
$$

From the variational formula given in $[\mathbf{6 0}],[\mathbf{1 0}]$ and $[\mathbf{7}]$, we have

$$
\frac{d}{d t} \mathcal{E}_{\nu}(g)=\int\left(\sigma_{2}(g)-\nu e^{4 u}\right) g^{-1} \cdot \frac{d}{d t} g d v o l(g)
$$

and

$$
\frac{d}{d t} \mathcal{F}_{1}(g)=\frac{n-2}{2} \int \sigma_{1}(g) g^{-1} \cdot \frac{d}{d t} \operatorname{gdvol}(g) .
$$


Now we introduce a Yamabe type flow, which non-increases $\mathcal{E}_{\nu}$ and preserves $\mathcal{F}_{1}$.

$$
\frac{d u}{d t}=-\frac{1}{2} g^{-1} \frac{d}{d t} g:=e^{-2 u} \frac{\sigma_{2}(g)-\nu e^{4 u}}{\sigma_{1}(g)}-r_{\nu}(g) e^{-2 u}+s_{\nu}(g),
$$

where $r_{\nu}(g)$ and $s_{\nu}(g)$ are space constants, given by

$$
r_{\nu}(g):=\frac{\mathcal{F}_{2}(g)-\int_{M} \nu e^{4 u} d v o l_{g}}{\mathcal{F}_{1}(g)}
$$

and

$$
\int_{M} \sigma_{1}(g)\left\{e^{-2 u} \frac{\sigma_{2}(g)-\nu e^{4 u}}{\sigma_{1}(g)}-r_{\nu}(g) e^{-2 u}+s_{\nu}(g)\right\} d v o l(g)=0 .
$$

Lemma 4. Flow (31) preserves $\mathcal{F}_{1}$ and non-increases $\mathcal{E}_{\nu}$. Hence when $n \geq 4$, then $r_{\nu}$ is non-increasing along the flow, and when $n=3$, then $r_{\nu}$ is non-decreasing along the flow.

Proof. By the definition of $s_{\nu}(g)$ and (30), flow (31) preserves $\mathcal{F}_{1}$. By the definition of $s_{\nu}$ and $r_{\nu}$, we can compute as follows

$$
\begin{array}{r}
\frac{d}{d t} \mathcal{E}_{\nu}(g)=\int_{M}\left(\sigma_{2}(g)-\nu e^{4 u}\right) g^{-1} \cdot \frac{d}{d t} g d v o l(g) \\
=-2 \int e^{2 u} \sigma_{1}(g)\left(e^{-2 u} \frac{\sigma_{2}(g)-\nu e^{4 u}}{\sigma_{1}(g)}-r_{\nu}(g) e^{-2 u}\right)^{2} d \operatorname{vol}(g) .
\end{array}
$$

Therefore, the desired result yields.

q.e.d.

\section{Local estimates}

In this section, we will establish a priori estimates for flow (31) and equations (8), (9) and (10). Local estimates for this class of fully nonlinear conformal equations were first given in $[\mathbf{2 6}]$. Since then there are many extensions. See for instance $[\mathbf{1 3}]$ and the survey paper [63]. It is important to note that the a priori estimates established below do not depend on the perturbation $\nu>0$.

Given $\nu>0$, assume $g_{0} \in \mathcal{C}_{1}\left(\left[g_{0}\right]\right)$. By Lemma 2, (31) is parabolic. By the standard implicit function theorem we have the short-time existence result. Let $T^{*} \in(0, \infty]$ so that $\left[0, T^{*}\right)$ is the maximum interval for the existence of the flow $g(t) \in \Gamma_{1}^{+}$.

Theorem 6. Assume that $n \geq 3, \nu>0$ and $g_{0} \in \Gamma_{1}^{+}$. Let $u$ be a solution of (31) in a geodesic ball $B_{R} \times[0, T]$ for $T<T^{*}$ and $R<\tau_{0}$, the injectivity radius of $M$.

(1) Assume that $\forall t \in[0, T]$

$$
r_{\nu}(t) \leq 0
$$


Then there is a constant $C$ depending only on $\left(B_{R}, g_{0}\right)$ (independent of $\nu$ and $T)$ such that for any $(x, t) \in B_{R / 2} \times[0, T]$

$$
|\nabla u|^{2}+\left|\nabla^{2} u\right| \leq C
$$

(2) Assume that $\forall t \in[0, T]$

$$
r_{\nu}(t)>0
$$

Then there is a constant $C$ depending only on $\left(B_{R}, g_{0}\right)$ (independent of $\nu$ and $T)$ such that for any $(x, t) \in B_{R / 2} \times[0, T]$

$$
|\nabla u|^{2}+\left|\nabla^{2} u\right| \leq C\left(1+\sup _{t \in[0, T]} r_{\nu}(t) \times e^{-2 \inf _{(x, t) \in B_{R} \times[0, T]} u(x, t)}\right) .
$$

In particular, if we assume $n \geq 4$, we have

$$
|\nabla u|^{2}+\left|\nabla^{2} u\right| \leq C\left(1+e^{-2 \inf _{(x, t) \in B_{R} \times[0, T]} u(x, t)}\right) .
$$

Proof. In the proof, $C$ is a constant independent of $T$ and $\nu$, which may vary from line to line. Let $W=\left(w_{i j}\right)$ be an $n \times n$ matrix with $w_{i j}=\nabla_{i j}^{2} u+u_{i} u_{j}-\frac{|\nabla u|^{2}}{2}\left(g_{0}\right)_{i j}+\left(S_{g_{0}}\right)_{i j}$. Here $u_{i}$ and $u_{i j}$ are the first and second derivatives of $u$ with respect to the background metric $g_{0}$. Recall $F_{\nu}(W)=\frac{\sigma_{2}(W)-\nu}{\sigma_{1}(W)}$. Set

$$
\begin{aligned}
\left(F_{\nu}^{i j}(W)\right) & :=\left(\frac{\partial F_{\nu}}{\partial w_{i j}}(W)\right) \\
& =\left(\frac{\sigma_{1}(W) T^{i j}-\sigma_{2}(W) \delta^{i j}+\nu \delta^{i j}}{\sigma_{1}^{2}(W)}\right)
\end{aligned}
$$

where $\left(T^{i j}\right)=\left(\sigma_{1}(W) \delta^{i j}-w^{i j}\right)$ is the first Newton transformation associated with $W$, and $\delta^{i j}$ is the Kronecker symbol. In view of Lemma 2 we know that $\left(F_{\nu}^{i j}\right)$ is positive definite and $F_{\nu}$ is concave in $\Gamma_{1}^{+}$. For the simplicity of notation, we now drop the index $\nu$, if there is no confusion. We try to show the local estimates for first and second order derivatives together. Let $S(T M)$ denote the unit tangent bundle of $M$ with respect to the background metric $g_{0}$. We define a function $\tilde{G}: S(T M) \times[0, T] \rightarrow \mathbb{R}$

$$
\tilde{G}(e, t)=\left(\nabla^{2} u+|\nabla u|^{2} g_{0}\right)(e, e)
$$


Without loss of generality, we assume $R=1$. Let $\rho \in C_{0}^{\infty}\left(B_{1}\right)$ be a cut-off function defined as in $[\mathbf{2 6}]$ such that

$$
\begin{aligned}
\rho & \geq 0, & & \text { in } B_{1}, \\
\rho & =1, & & \text { in } B_{1 / 2}, \\
|\nabla \rho(x)| & \leq 2 b_{0} \rho^{1 / 2}(x), & & \text { in } B_{1}, \\
\left|\nabla^{2} \rho\right| & \leq b_{0}, & & \text { in } B_{1} .
\end{aligned}
$$

Here $b_{0}>1$ is a constant. Since $e^{-2 u} g_{0} \in \Gamma_{1}^{+}$, to bound $|\nabla u|$ and $\left|\nabla^{2} u\right|$ we only need to bound $\left(\nabla^{2} u+|\nabla u|^{2} g_{0}\right)(e, e)$ from above for all $e \in S(T M)$ and for all $t \in[0, T]$. For this purpose, denote $G(e, t)=$ $\rho(x) \tilde{G}(e, t)$. Assume $\left(e_{1}, t_{0}\right) \in S\left(T_{x_{0}} M\right) \times[0, T]$ such that

$$
\begin{array}{r}
G\left(e_{1}, t_{0}\right)=\max _{S(T M) \times[0, T]} G(e, t), \\
t_{0}>0, \\
G\left(e_{1}, t_{0}\right)>n \max _{B_{1}} \sigma_{1}\left(g_{0}\right) .
\end{array}
$$

Let $\left(e_{1}, \cdots, e_{n}\right)$ be a orthonormal basis at point $\left(x_{0}, t_{0}\right)$. It follows from the fact $W \in \Gamma_{1}^{+}$

$$
\begin{aligned}
n G\left(e_{1}, t_{0}\right) & \geq \rho\left(\Delta u+n|\nabla u|^{2}\right) \geq \rho\left(n|\nabla u|^{2}+\frac{n-2}{2}|\nabla u|^{2}-\sigma_{1}\left(g_{0}\right)\right), \\
& \geq \frac{3 n-2}{2} \rho|\nabla u|^{2}-\frac{1}{n} G\left(e_{1}, t_{0}\right),
\end{aligned}
$$

so that

$$
G\left(e_{1}, t_{0}\right) \geq \frac{\frac{3 n-2}{2}}{n+\frac{1}{n}} \rho|\nabla u|^{2} \geq \frac{21}{20} \rho|\nabla u|^{2} .
$$

Consequently, we obtain

$$
\nabla_{11}^{2} u\left(x_{0}, t_{0}\right) \geq \frac{1}{20}|\nabla u|^{2}\left(x_{0}, t_{0}\right) .
$$

Set for any $i \neq j=1, \cdots, n$

$$
e^{\prime}=\frac{1}{\sqrt{2}}\left(e_{i} \pm e_{j}\right)
$$

We have

$$
G\left(e^{\prime}, t_{0}\right)=\frac{1}{2}\left(G\left(e_{i}, t_{0}\right)+G\left(e_{j}, t_{0}\right)\right) \pm \rho \nabla_{i j}^{2} u\left(x_{0}, t_{0}\right) .
$$

Thus, there holds

$$
\rho\left|\nabla_{i j}^{2} u\left(x_{0}, t_{0}\right)\right| \leq G\left(e_{1}, t_{0}\right)-\frac{1}{2}\left(G\left(e_{i}, t_{0}\right)+G\left(e_{j}, t_{0}\right)\right) .
$$

On the other hand, we have $\forall i=1, \cdots, n$

$$
(n-1) G\left(e_{1}, t_{0}\right)+G\left(e_{i}, t_{0}\right) \geq \rho\left(\Delta u+n|\nabla u|^{2}\right) \geq
$$




$$
\rho\left(\frac{3 n-2}{2}|\nabla u|^{2}-\sigma_{1}\left(g_{0}\right)\right)
$$

which implies

$$
G\left(e_{i}, t_{0}\right) \geq \rho\left(\frac{3 n-2}{2}|\nabla u|^{2}-\sigma_{1}\left(g_{0}\right)\right)-(n-1) G\left(e_{1}, t_{0}\right) .
$$

Together with (46), we deduce

$\rho\left|\nabla_{i j}^{2} u\left(x_{0}, t_{0}\right)\right| \leq n G\left(e_{1}, t_{0}\right)-\frac{3 n-2}{2} \rho|\nabla u|^{2}+\rho \sigma_{1}\left(g_{0}\right) \leq(n+1) G\left(e_{1}, t_{0}\right)$.

(Indeed, at any point $(x, t)$, the estimate $\rho\left|\nabla_{i j}^{2} u\right| \leq(n+1) G\left(e_{1}, t_{0}\right)$ holds). Now choose the normal coordinates around $x_{0}$ such that at point $x_{0}$

$$
\frac{\partial}{\partial x_{1}}=e_{1}
$$

and consider the function $G$ on $M \times[0, T]$ defined by

$$
G(x, t):=\rho(x)\left(u_{11}+|\nabla u|^{2}\right)(x, t) .
$$

Clearly, $\left(x_{0}, t_{0}\right)$ is a maximum point of $G(x, t)$ on $M \times[0, T]$. At $\left(x_{0}, t_{0}\right)$, we have

$$
\begin{aligned}
& 0 \leq G_{t}=\rho\left(u_{11 t}+2 \sum_{l} u_{l} u_{l t}\right), \\
& 0=G_{j}=\frac{\rho_{j}}{\rho} G+\rho\left(u_{11 j}+2 \sum_{l \geq 1} u_{l} u_{l j}\right), \quad \text { for any } j,
\end{aligned}
$$

$$
\begin{aligned}
& 0 \geq \\
& \left(G_{i j}\right)=\left(\frac{\rho \rho_{i j}-2 \rho_{i} \rho_{j}}{\rho^{2}} G+\rho\left(u_{11 i j}+\sum_{l \geq 1}\left(2 u_{l i} u_{l j}+2 u_{l} u_{l i j}\right)\right)\right) .
\end{aligned}
$$

Recall that $\left(F^{i j}\right)$ is definite positive. Hence, we have

$$
\begin{aligned}
0 \geq & \sum_{i, j \geq 1} F^{i j} G_{i j}-G_{t} \\
\geq & \sum_{i, j \geq 1} F^{i j} \frac{\rho \rho_{i j}-2 \rho_{i} \rho_{j}}{\rho^{2}} G \\
& +\rho \sum_{i, j \geq 1} F^{i j}\left(u_{11 i j}+\sum_{l \geq 1}\left(2 u_{l i} u_{l j}+2 u_{l} u_{l i j}\right)\right) \\
& \quad-\rho\left(u_{11 t}+2 \sum_{l \geq 1} u_{l} u_{l t}\right) .
\end{aligned}
$$


First, from the definition of $\rho$, we have

$$
\sum_{i, j \geq 1} F^{i j} \frac{\rho \rho_{i j}-2 \rho_{i} \rho_{j}}{\rho^{2}} G \geq-C \sum_{i, j \geq 1}\left|F^{i j}\right| \frac{1}{\rho} G,
$$

and

$$
\begin{aligned}
\sum_{i, j \geq 1}\left|F^{i j}\right| & \geq \sum_{i} F^{i i} \\
& =\left(n-1-\frac{n \sigma_{2}(W)}{\sigma_{1}^{2}(W)}\right)+\frac{n \nu}{\sigma_{1}^{2}(W)} \geq C \sum_{i, j \geq 1}\left|F^{i j}\right|
\end{aligned}
$$

since $W$ is positive definite . Using the facts that

$$
u_{k i j}=u_{i j k}+\sum_{m} R_{m i k j} u_{m}
$$

$$
u_{k k i j}=
$$

$u_{i j k k}+\sum_{m}\left(2 R_{m i k j} u_{m k}-R i c_{m j} u_{m i}-R i c_{m i} u_{m j}-R i c_{m i, j} u_{m}+R_{m i k j, k} u_{m}\right)$

and

$$
\left(\sum_{l} u_{l}^{2}\right)_{11}=2 \sum_{l}\left(u_{11 l} u_{l}+u_{1 l}^{2}\right)+O\left(|\nabla u|^{2}\right),
$$

we have

$$
\begin{aligned}
& \sum_{i, j \geq 1} F^{i j} u_{11 i j} \\
\geq & \sum_{i, j \geq 1} F^{i j}\left(w_{i j 11}-\left(u_{11}\right)_{i} u_{j}-u_{i}\left(u_{11}\right)_{j}+\sum_{l \geq 1}\left(u_{1 l}^{2}+u_{11 l} u_{l}\right)\left(g_{0}\right)_{i j}\right) \\
& -2 \sum_{i, j \geq 1} F^{i j} u_{i 1} u_{j 1}-C\left(1+\left|\nabla^{2} u\right|+|\nabla u|^{2}\right) \sum_{i, j \geq 1}\left|F^{i j}\right|
\end{aligned}
$$

and

(60)

$$
\begin{aligned}
& \sum_{i, j, l} F^{i j} u_{l} u_{l i j} \\
\geq & \sum_{i, j, l} F^{i j} u_{l} w_{i j l}-\sum_{i, j, l} F^{i j}\left(u_{l} u_{i l} u_{j}+u_{l} u_{i} u_{j l}\right) \\
& +\frac{1}{2} \sum_{i, j} F^{i j}\left\langle\nabla u, \nabla\left(|\nabla u|^{2}\right)\right\rangle\left(g_{0}\right)_{i j}-C\left(1+|\nabla u|^{2}\right) \sum_{i, j \geq 1}\left|F^{i j}\right| .
\end{aligned}
$$


Combining (59) and (60), we deduce

(61)

$$
\begin{aligned}
& \sum_{i, j \geq 1} F^{i j}\left(u_{11 i j}+2 \sum_{l \geq 1}\left(u_{l i} u_{l j}+u_{l} u_{l i j}\right)\right) \\
\geq & \sum_{i, j \geq 1} F^{i j}\left(w_{i j 11}+2 \sum_{l \geq 1} w_{i j l} u_{l}\right) \\
& +2 \sum_{i, j \geq 1} F^{i j} \sum_{l \geq 2} u_{l i} u_{l j}+\sum_{i, j, l \geq 1} u_{1 l}^{2} F^{i j}\left(g_{0}\right)_{i j} \\
& -\sum_{i, j} F^{i j}\left[\left(u_{11}+|\nabla u|^{2}\right)_{i} u_{j}+u_{i}\left(u_{11}+|\nabla u|^{2}\right)_{j}\right. \\
& \left.-\left\langle\nabla u, \nabla\left(u_{11}+|\nabla u|^{2}\right)\right\rangle\left(g_{0}\right)_{i j}\right]-C\left(1+\left|\nabla^{2} u\right|+|\nabla u|^{2}\right) \sum_{i, j \geq 1}\left|F^{i j}\right| \\
\geq & \sum_{i, j} F^{i j}\left(w_{i j 11}+2 \sum_{l} w_{i j l} u_{l}\right)+u_{11}^{2} \sum_{i, j} F^{i j}\left(g_{0}\right)_{i j} \\
& +\sum_{i, j} F^{i j}\left(\rho_{i} u_{j}+\rho_{j} u_{i}-\langle\nabla \rho, \nabla u\rangle\left(g_{0}\right)_{i j}\right) \frac{G}{\rho^{2}} \\
& -C\left(1+\left|\nabla^{2} u\right|+|\nabla u|^{2}\right) \sum_{i, j \geq 1}\left|F^{i j}\right| .
\end{aligned}
$$

Now, we want to estimate $\sum_{i, j, l} F^{i j} w_{i j l} u_{l}$ and $\sum_{i, j} F^{i j} w_{i j 11}$ respectively. Using Lemma 1, there holds

$$
\sum_{i, j, l} F^{i j} w_{i j l} u_{l}=\sum_{l} F_{l} u_{l}
$$

and

$$
\sum_{i, j} F^{i j} w_{i j 11}=F_{11}-\sum_{i, j, k, m} \frac{\partial^{2} F}{\partial w_{i j} \partial w_{k m}} w_{i j 1} w_{k m 1} \geq F_{11} .
$$

Therefore, these estimates give

$$
\sum_{i, j \geq 1} F^{i j}\left(w_{i j 11}+2 \sum_{l \geq 1} w_{i j l} u_{l}\right) \geq F_{11}+2 \sum_{l} F_{l} u_{l} .
$$

Recall from (31) that

$$
F=u_{t}+r_{\nu}(g) e^{-2 u}-s_{\nu}(g)
$$

so that

$$
F_{11}=u_{11 t}+r_{\nu}(g) e^{-2 u}\left(-2 u_{11}+4 u_{1}^{2}\right)
$$




$$
F_{l}=u_{l t}+r_{\nu}(g) e^{-2 u}\left(-2 u_{l}\right), \forall l=1, \cdots, n .
$$

Gathering (44), (49), (53), (54), (55), (61) (64), (66) and (67), we obtain

$$
\begin{aligned}
0 \geq & -C\left(\sum_{i, j}\left|F^{i j}\right|\right) \frac{G}{\rho}+\rho\left(\sum_{i} F^{i i}\right) u_{11}^{2} \\
& -C \rho\left(\sum_{i, j}\left|F^{i j}\right|\right)\left(1+|\nabla u|^{2}+\left|\nabla^{2} u\right|\right) \\
& +\sum_{i, j} F^{i j}\left(\rho_{i} u_{j}+\rho_{j} u_{i}-\langle\nabla \rho, \nabla u\rangle\left(g_{0}\right)_{i j}\right) \frac{G}{\rho} \\
& +\rho r_{\nu}(g) e^{-2 u}\left(-2 u_{11}-4 \sum_{l=2}^{n} u_{l}^{2}\right) \\
\geq & -C\left(\sum_{i} F^{i i}\right) \frac{G}{\rho}+\rho\left(\sum_{i} F^{i i}\right) u_{11}^{2} \\
& -C\left(\sum_{i} F^{i i}\right) \frac{G|\nabla u|}{\sqrt{\rho}}-C\left(\sum_{i} F^{i i}\right)(\rho+G),
\end{aligned}
$$

since $r_{\nu}(g) \leq 0$ for any $t \in[0, T]$. As a consequence, there holds

$$
C \rho^{2} \geq-C G-C G \sqrt{G}+\rho^{2} u_{11}^{2} .
$$

Recall (44) holds at point $\left(x_{0}, t_{0}\right)$ so that

$$
G\left(x_{0}, t_{0}\right)=\rho\left(u_{11}+|\nabla u|^{2}\right)\left(x_{0}, t_{0}\right) \leq 21\left(\rho u_{11}\right)\left(x_{0}, t_{0}\right) .
$$

Together with (69), we deduce

$$
C \rho^{2} \geq-C G-C G \sqrt{G}+\frac{G^{2}}{21^{2}}
$$

which implies

$$
C \geq-C G+\frac{G^{2}}{2 \times 21^{2}}
$$

This yields

$$
G \leq C
$$

Here $C$ is a constant independent of $T$ and $\nu$. Therefore, we have finished the proof of the first part of the Theorem. The second part yields from (68) and Lemma 4.

q.e.d.

The same proof gives the local estimates for the elliptic equation.

Corollary 4. Assume $n \geq 3, \nu>0$ and $g_{0} \in \Gamma_{1}^{+}$. Let $B_{R}$ be a geodesic ball for $R<\tau_{0}$, the injectivity radius of $M$. Assume that 
$e^{-2 u} g_{0} \in \Gamma_{1}^{+}$is a solution of the following equation in $B_{R}$

$$
\frac{\sigma_{2}(g)-\nu e^{4 u}}{\sigma_{1}(g)}=\kappa
$$

for some constant $\kappa$.

(1) If $\kappa \leq 0$, then there is a constant $C$ depending only on $\left(B_{R}, g_{0}\right)$ (independent of $\nu$ ) such that for any $x \in B_{R / 2}$

$$
|\nabla u|^{2}+\left|\nabla^{2} u\right| \leq C \text {. }
$$

(2) If $\kappa>0$, then there is a constant $C$ depending only on $\left(B_{R}, g_{0}\right)$ and $\kappa$ (independent of $\nu$ ) such that for any $x \in B_{R / 2}$

$$
|\nabla u|^{2}+\left|\nabla^{2} u\right| \leq C\left(1+e^{-2 \inf _{x \in B_{R}} u(x)}\right) .
$$

Corollary 5. Under the same hypotheses as in Theorem 6 with $r_{\nu} \leq$ 0 there is a constant $C$ depending only on $g_{0}$ (independent of $\nu$ ) such that for any $t \in\left[0, T^{*}\right)$

$$
\|\nabla u\|_{C(M)}+\left\|\nabla^{2} u\right\|_{C(M)} \leq C .
$$

Corollary 6. Under the same hypotheses as in Theorem 6 with $r_{\nu} \leq$ 0 , then there is a constant $C$ depending only on $g_{0}$ (independent of $\nu$ ) such that for any $t \in\left[0, T^{*}\right)$

$$
\|u\|_{C^{2}(M)} \leq C
$$

Proof. First, for any $t \in\left[0, T^{*}\right)$ we have

$$
\mathcal{F}_{1}(g(t)) \equiv \mathcal{F}_{1}(g(0))
$$

Hence the following Sobolev inequality

$$
\int_{M} \sigma_{1}(g) \operatorname{dvol}(g) \geq Y_{1}\left(\left[g_{0}\right]\right)(\operatorname{Vol}(g))^{\frac{n-2}{n}}, \forall g \in \Gamma_{1}^{+},
$$

implies that the volume $\operatorname{Vol}(g(t))$ along the flow is bounded from above, i.e.,

$$
\int_{M} e^{-n u} d v o l\left(g_{0}\right)<C,
$$

for some constant $C>0$. On the other hand, applying Corollary 5, we have

$$
\int_{M} e^{2 u} d \operatorname{vol}(g) \geq C \int_{M} \sigma_{1}(g) \operatorname{dvol}(g)=C \mathcal{F}_{1}(g(0))>0 .
$$

This, together with (80), Hölder's inequality and Corollary 5, implies $u$ is uniformly bounded. In view of Corollary 5, we prove the result.

q.e.d. 
We remark that in this paper we only use (1) of Theorem 6. (2) of Theorem 6 will be used in a forthcoming paper.

\section{Proof of Theorems 1 and 2}

Now for $\nu>0$ we define

$$
a_{\nu}:= \begin{cases}\inf _{g \in \mathcal{C}_{1}\left(\left[g_{0}\right]\right)} \frac{\mathcal{E}_{\nu}(g)}{\left(\int_{M} \sigma_{1}(g) \operatorname{dvol}(g)\right)^{\frac{n-4}{n-2}}} & \text { if } n \neq 4 \\ \int_{M} \sigma_{2}(g) \operatorname{dvol}(g)-\nu & \text { if } n=4 ;\end{cases}
$$

If $n \neq 4$ and $a_{\nu}$ is achieved by a metric $g=e^{-2 u} g_{0}$, the $g$ satisfies

$$
\frac{\sigma_{2}(g)-\nu e^{4 u}}{\sigma_{1}(g)}=\kappa
$$

for some constant $\kappa$. Equivalently, we will consider the energy functional $\mathcal{E}_{\nu}$ on the normalized cone $\tilde{\mathcal{C}}_{1}\left(\left[g_{0}\right]\right)$

$$
\tilde{\mathcal{C}}_{1}\left(\left[g_{0}\right]\right):=\left\{g \in \mathcal{C}_{1}\left(\left[g_{0}\right]\right) \mid \int_{M} \sigma_{1}(g) \operatorname{dvol}(g)=1\right\} .
$$

The first step is to solve the perturbed equation (82).

Proposition 1. Let $\nu>0$. In the following three cases

1) when $n \geq 5, a_{\nu}<0$,

2) when $n=4, a_{\nu}<0$, which is equivalent to $\int_{M} \sigma_{2}(g) d v o l(g)-\nu<$ 0 ,

3) when $n=3, a_{\nu}>0$,

flow (31) globally converges to a solution of (82). As a direct application, $a_{\nu}$ is achieved by a function $u_{\nu}$ satisfying (82) for $\kappa=-1$. Moreover, such a solution is unique.

Proof. The proof follows closely the proof given in [18]. When $n \geq 5$, without loss of generality we choose $g_{0} \in \Gamma_{1}^{+}$such that $\mathcal{E}_{\nu}\left(g_{0}\right)<0$ and $\int_{M} \sigma_{1}\left(g_{0}\right) d \operatorname{vol}\left(g_{0}\right)=1$. Using Lemma 4, we have $r_{\nu}(g(t)) \leq r_{\nu}(g(0))<$ $0, \forall t \in\left[0, T^{*}\right)$. If $n=3$, or $n=4$, by the hypotheses, we have $r_{\nu}(g(t))<$ $-c<0, \forall t \in\left[0, T^{*}\right)$. Applying Corollary 6, the solution $u$ of flow (31) has a uniform $C^{2}$ bound, which is independent of $t$. We divide the proof into 3 steps.

Step 1. The flow preserves the positivity of the scalar curvature. This is another crucial point of this paper. 
Proposition 2. There is a constant $C_{0}>0$, independent of $T \in$ $\left[0, T^{*}\right)$ and $\nu$ such that $\sigma_{1}(g(t))>C_{0} \nu$ for any $t \in[0, T]$.

Proof. The proof follows closely the proof given in $[\mathbf{2 7}]$ and $[\mathbf{1 8}]$. Recall

$$
\begin{aligned}
& W=\left(w_{i j}\right)=\left(\nabla_{i j}^{2} u+u_{i} u_{j}-\frac{|\nabla u|^{2}}{2}\left(g_{0}\right)_{i j}+\left(S_{g_{0}}\right)_{i j}\right), \\
& F_{\nu}(W)=\frac{\sigma_{2}(W)-\nu}{\sigma_{1}(W)} .
\end{aligned}
$$

Hence, $F_{\nu}=u_{t}+r_{\nu}(g(t)) e^{-2 u}-s_{\nu}(g(t))$. Without loss of generality, we assume that the minimum of $F_{\nu}$ is achieved at $\left(x_{0}, t_{0}\right) \in M \times(0, T]$. Near $\left(x_{0}, t_{0}\right)$, we have

$$
\frac{d}{d t} F_{\nu}=\sum_{i j} A^{i j}\left(\nabla_{g}^{2}\left(u_{t}\right)\right)_{i j}=\sum_{i j} A^{i j}\left[\left(\nabla_{g}^{2}\left(F_{\nu}\right)\right)_{i j}-r_{\nu}(g)\left(\nabla_{g}^{2}\left(e^{-2 u}\right)\right)_{i j}\right],
$$

where

$$
A^{i j}:=\frac{\partial F_{\nu}}{\partial w_{i j}}=\frac{\left(\sigma_{1}^{2}(W)-\sigma_{2}(W)+\nu\right) \delta^{i j}-\sigma_{1}(W) W^{i j}}{\sigma_{1}^{2}(W)}
$$

is positive definite. To simplify the notation, we drop the index $\nu$ as before. Since $\left(x_{0}, t_{0}\right)$ is the minimum of $F$ in $M \times[0, T]$, at this point, we have $\frac{d F}{d t} \leq 0, F_{l}=0 \quad \forall l$ and $\left(F_{i j}\right)$ is non-negative definite. Note that

$$
\left(\nabla_{g}^{2}\right)_{i j} F=F_{i j}+u_{i} F_{j}+u_{j} F_{i}-\sum_{l} u_{l} F_{l} \delta_{i j}=F_{i j}
$$

at $\left(x_{0}, t_{0}\right)$, where $F_{j}$ and $F_{i j}$ are the first and second derivatives with respect to the back-ground metric $g_{0}$. From the positivity of $A$ and (84), we have

$$
\begin{gathered}
0 \geq F_{t}-\sum_{i, j} A^{i j} F_{i j} \\
\geq-r_{\nu}(g) \sum_{i, j} A^{i j}\left\{\left(e^{-2 u}\right)_{i j}+u_{i}\left(e^{-2 u}\right)_{j}+u_{j}\left(e^{-2 u}\right)_{i}-\sum_{l} u_{l}\left(e^{-2 u}\right)_{l} \delta_{i j}\right\} \\
=-r_{\nu}(g) e^{-2 u} \sum_{i, j} A^{i j}\left\{-2 w_{i j}+2 u_{i} u_{j}+2 S\left(g_{0}\right)_{i j}+|\nabla u|^{2} \delta_{i j}\right\} \\
\geq-r_{\nu}(g) e^{-2 u}\left(\frac{-2 \sigma_{2}(W)-2 \nu}{\sigma_{1}(W)}\right) \\
-r_{\nu}(g) e^{-2 u} \sum_{i, j} A^{i j}\left(2 u_{i} u_{j}+2 S\left(g_{0}\right)_{i j}+|\nabla u|^{2} \delta_{i j}\right) .
\end{gathered}
$$


Here we have used $\sum_{i, j} A^{i j} w_{i j}=\frac{\sigma_{2}(W)+\nu}{\sigma_{1}(W)}$. On the other hand, we have

$$
\begin{aligned}
& \sum_{i, j} A^{i j} S\left(g_{0}\right)_{i j} \\
= & \frac{\left(\sigma_{1}^{2}(W)-\sigma_{2}(W)\right) \sigma_{1}\left(g_{0}\right)}{\sigma_{1}^{2}(W)}-\frac{1}{\sigma_{1}(W)} \sum_{i, j} W^{i j} S\left(g_{0}\right)_{i j}+\frac{\nu \sigma_{1}\left(g_{0}\right)}{\sigma_{1}^{2}(W)} .
\end{aligned}
$$

Going back to (85), we have

$$
\begin{aligned}
0 \geq & F_{t}-\sum_{i, j} A^{i j} F_{i j} \\
\geq & -r_{\nu}(g) e^{-2 u}\left[\frac{-2 \sigma_{2}(W)-2 \nu}{\sigma_{1}(W)}+\frac{2\left(\sigma_{1}^{2}(W)-\sigma_{2}(W)\right) \sigma_{1}\left(g_{0}\right)}{\sigma_{1}^{2}(W)}\right. \\
& \left.\quad-\frac{2}{\sigma_{1}(W)} \sum_{i, j} W^{i j} S\left(g_{0}\right)_{i j}+\frac{2 \nu \sigma_{1}\left(g_{0}\right)}{\sigma_{1}^{2}(W)}\right]
\end{aligned}
$$

since $\left(A^{i j}\right)$ is positive definite and $r_{\nu}(g)<-c$ is negative. Let us use $O(1)$ denote terms with a uniform bound. One can check $\sigma_{2}(W)=O(1)$ for $\|u\|_{C^{2}}$ is uniformly bounded and $\sum_{i, j} W^{i j} S\left(g_{0}\right)_{i j}=O(1)$. Also the term $\sigma_{1}^{2}(W)-\sigma_{2}(W)$ is always non-negative. From (87), we conclude that there is a positive constant $C_{2}>0$ (independent of $T$ and $\nu$ ) such that

$$
\frac{\left|\sigma_{2}(W)\left(x_{0}, t_{0}\right)\right|+\nu}{\sigma_{1}(W)\left(x_{0}, t_{0}\right)}<C_{2} .
$$

Since $(x, t)$ is the minimum of $F_{\nu}(W)$ in $M \times[0, T]$, for any $(x, t) \in$ $M \times[0, T]$ we have

$$
\frac{\sigma_{2}(W)(x, t)-\nu}{\sigma_{1}(W)(x, t)} \geq-C_{2} .
$$

Hence, there is a positive constant $C>0$, independent of $T$ and $\nu$, such that

$$
\sigma_{1}(W)(x, t) \geq C \nu,
$$

for $\sigma_{2}(W) \leq \frac{1}{2} \sigma_{1}^{2}(W)$ provided $\sigma_{2}(W) \geq 0$. This finishes the proof of the Proposition and this step.

q.e.d.

Step 2. Now we can prove equation (82) admits a solution. From Step 1, we know that the flow is uniformly parabolic. And, Krylov's theory implies that $u(t)$ has a uniform $C^{2, \alpha}$ bound. Hence, $T^{*}=\infty$. 
One can also show that $u(t)$ globally converges to $u(\infty)$, which clearly is a solution of (82) for $k=r_{\nu}(g(\infty))$. (Note that $r_{\nu}(g(t))$ is monotone and bounded so that $r_{\nu}(g(\infty))$ exists) (see $[\mathbf{2 7}]$ ). So $u_{\nu}=u(\infty)-$ $\frac{1}{2} \log \left|r_{\nu}(g(\infty))\right|$ solves (82) for $\kappa=-1$.

Step 3. The solution to equation (82) for $k=-1$ is unique.

Assume $u_{1}$ and $u_{2}$ are two solutions. We consider the function $v=$ $u_{1}-u_{2}$, which solves the following second order elliptic equation

$$
\sum_{i, j} A^{i j}(x) v_{i j}+\sum_{i} B^{i} v_{i}+m(x) v=0
$$

where

$$
A^{i j}(x)=\int_{0}^{1} F^{i j}\left(t W_{2}+(1-t) W_{1}\right) d t
$$

$W_{l}(x)=\left(\left(u_{l}\right)_{i j}+\left(u_{l}\right)_{i}\left(u_{l}\right)_{j}-\frac{1}{2}\left|\nabla u_{l}\right|^{2}\left(g_{0}\right)_{i j}+\left(S\left(g_{0}\right)\right)_{i j}\right), \quad$ for $l=1,2$

and

$$
m(x)=-2 \int_{0}^{1} e^{-2\left((1-t) u_{1}(x)+t u_{2}(x)\right)} d t<0 .
$$

This is a uniformly elliptic equation. Now applying the classical strong maximum principle, we deduce that

$$
v \equiv 0 .
$$

Hence we have the uniqueness. It is clear that the unique solution achieves $a_{\nu}$. Thus, we finish the proof.

q.e.d.

In the following, we study a nonlinear eigenvalue problem for the operator

$$
\sigma_{2}\left(\nabla^{2} u+d u \otimes d u-\frac{|\nabla u|^{2}}{2} g_{0}+S_{g_{0}}\right)
$$

in $\Gamma_{1}^{+}$. The nonlinear eigenvalue problem for the operator (90) in $\Gamma_{2}^{+}$ was considered in the first version of [27]. In that paper, the nonlinear eigenvalue problem can only be considered in $\Gamma_{2}^{+}$. With our analysis established for $\sigma_{2} / \sigma_{1}$, we can consider the nonlinear eigenvalue problem for the operator (90) in a larger class $\Gamma_{1}^{+}$.

Proposition 3. Let $\left(M^{n}, g_{0}\right)$ be a compact Riemannian manifold with $g_{0} \in \Gamma_{1}^{+}$and $n \geq 3$. Assume that the first eigenvalue $\lambda\left(g_{0}, \sigma_{2}\right)>0$. Then $\mathcal{C}_{2}\left(\left[g_{0}\right]\right)$ is not empty. Namely there exists a metric in $\left[g_{0}\right]$ with

$$
\sigma_{1}(g)>0 \text { and } \sigma_{2}(g)>0 .
$$


Moreover, there is a regular metric $g=e^{-2 u} g_{0} \in \mathcal{C}_{2}\left(\left[g_{0}\right]\right)$ satisfying

$$
\sigma_{2}(g)=\lambda e^{4 u},
$$

for $\lambda=\lambda\left(g_{0}, \sigma_{2}\right)>0$.

Proof. We define a function $h$

$$
h:(0,+\infty) \rightarrow \mathbb{R}= \begin{cases}\inf _{g \in \mathcal{C}_{1}\left(\left[g_{0}\right]\right)} \frac{\mathcal{E}_{\nu}(g)}{\left(\int_{M} \sigma_{1}(g) \operatorname{dvol}(g)\right)^{\frac{n-4}{n-2}}} & \text { if } n \neq 4 ; \\ \int_{M} \sigma_{2}(g) \operatorname{dvol}(g)-\nu & \text { if } n=4 ;\end{cases}
$$

Thus, $h$ is a non-increasing function if $n \geq 4$ and a non-decreasing function if $n=3$. First, we consider the case $n \geq 4$. Define a set $A$

$$
A:=\{\nu \in(0,+\infty) \mid h(\nu)<0\} .
$$

By the assumption that $\lambda\left(g_{0}, \sigma_{2}\right)>0$, it is easy to check that

$$
\left(\lambda\left(g_{0}, \sigma_{2}\right),+\infty\right) \subset A \text { and }\left(0, \lambda\left(g_{0}, \sigma_{2}\right)\right) \cap A=\emptyset .
$$

Hence for any $\nu>\lambda\left(g_{0}, \sigma_{2}\right)$ we have $h(\nu)<0$. By Proposition 1 we have a smooth metric $g_{u_{\nu}}=e^{-2 u_{\nu}} g_{0} \in \mathcal{C}_{1}\left(\left[g_{0}\right]\right)$, which solves equation (82) for $\kappa=h(\nu)$ and satisfies $\int_{M} \sigma_{1}\left(g_{u_{\nu}}\right) \operatorname{dvol}\left(g_{u_{\nu}}\right)=1$. From Corollary 6 , the set of solutions

$$
\left\{u_{\nu}, \forall \nu \in\left(\lambda\left(g_{0}, \sigma_{2}\right), \lambda\left(g_{0}, \sigma_{2}\right)+1\right)\right\}
$$

is uniformly bounded in $C^{2}$ norm. From equation (82), $\sigma_{1}\left(g_{u_{\nu}}\right)$ is uniformly bounded from below by a positive constant for $\nu \in\left(\lambda\left(g_{0}, \sigma_{2}\right)\right.$, $\left.\lambda\left(g_{0}, \sigma_{2}\right)+1\right)$, since $\nu>\lambda\left(g_{0}, \sigma_{2}\right)>0$ and also we have $\frac{\sigma_{2}(W)}{\sigma_{1}(W)} \leq$ $\frac{1}{2} \sigma_{1}(W)$, provided that $\sigma_{1}(W)>0$. By the Krylov's result, the set of solutions $\left\{u_{\nu}, \forall \nu \in\left(\lambda\left(g_{0}, \sigma_{2}\right), \lambda\left(g_{0}, \sigma_{2}\right)+1\right)\right\}$ is also uniformly bounded in $C^{2, \alpha}$ for $\alpha>0$. Define

$$
\kappa_{0}:=\lim _{\nu \rightarrow \lambda\left(g_{0}, \sigma_{2}\right)^{+}} h(\nu) .
$$

When $\nu \rightarrow \lambda\left(g_{0}, \sigma_{2}\right), u_{\nu}$ (by taking a subsequence) converges in $C^{2}$ to $u_{0} \in \Gamma_{1}^{+}$, which is a solution of (82) for $\nu=\lambda\left(g_{0}, \sigma_{2}\right)$ and $\kappa=\kappa_{0}$. Clearly, $\kappa_{0} \leq 0$. We claim

$$
\kappa_{0}=0 .
$$

Otherwise, $\mathcal{E}_{\lambda\left(g_{0}, \sigma_{2}\right)}\left(u_{0}\right)<0$, which implies that there is some small $\varepsilon>0$ such that $\forall \nu \in\left(\lambda\left(g_{0}, \sigma_{2}\right)-\varepsilon, \lambda\left(g_{0}, \sigma_{2}\right)\right]$, we have $\mathcal{E}_{\nu}\left(u_{0}\right)<0$. Hence $h(\nu)<0$ for all $\nu \in\left(\lambda\left(g_{0}, \sigma_{2}\right)-\varepsilon,+\infty\right)$, that is, $\left(\lambda\left(g_{0}, \sigma_{2}\right)-\varepsilon,+\infty\right) \subset A$. 
This contradicts to (93). Hence we have (95), which is equivalent to say that

$$
\sigma_{2}\left(g_{u_{0}}\right)-\lambda\left(g_{0}, \sigma_{2}\right) e^{4 u_{0}}=0 .
$$

This means that $u_{0} \in \Gamma_{2}^{+}$solves (91) for $\lambda=\lambda\left(g_{0}, \sigma_{2}\right)$. In particular, $u_{0} \in \Gamma_{2}^{+}$.

The proof for the case $n=3$ is similar by consider a set $A$ defined by

$$
A:=\{\nu \in(0,+\infty) \mid h(\nu)>0\} .
$$

We leave the proof to the interested reader.

q.e.d.

Remark 4. In the case $n \geq 4$, the function $h(\nu)$ is Lipschitz continuous. Indeed, for all $\nu^{\prime}<\nu$ and for all $g \in \mathcal{C}_{1}\left(\left[g_{0}\right]\right)$, we have

$$
\begin{aligned}
0 & <\mathcal{E}_{\nu^{\prime}}(u)-\mathcal{E}_{\nu}(u)=\left(\nu-\nu^{\prime}\right) \int e^{4 u} d \operatorname{vol}(g) \leq c\left(\nu-\nu^{\prime}\right)(\operatorname{Vol}(g))^{\frac{n-4}{n}} \\
& \leq c Y_{1}([g])^{\frac{4-n}{n-2}}\left(\nu-\nu^{\prime}\right)\left(\int \sigma_{1}(g) \operatorname{dvol}(g)\right)^{\frac{n-4}{n-2}}
\end{aligned}
$$

This proves the claim.

Proof of Theorem 2. First we discuss the case $Y_{2,1}\left(\left[g_{0}\right]\right)>0$. Using Lemma 3 , we have $\lambda\left(g_{0}, \sigma_{2}\right)>0$. By Proposition 3 , the cone $\Gamma_{2}^{+}$is not empty. Hence, from the results in [19] for the cases $n \geq 5$, in [11] for $n=4$ and $[\mathbf{3 3}]$ for the case $n=3$, there is a solution of (8).

Now suppose $Y_{2,1}\left(\left[g_{0}\right]\right)=0$. As in the proof of Proposition 3, we consider the function $h(\nu)$ for all $\nu>0$. With the same arguments, for any small positive $\nu>0$, equation (82) admits the unique solution $u_{\nu}$, since $h(\nu)<0 \forall \nu>0$ if $n \geq 4$ and $h(\nu)>0 \forall \nu>0$ if $n=3$. This fact is clear for $n \geq 4$. And in the case $n=3$, we have

$$
\inf _{g \in \mathcal{C}_{1}\left(\left[g_{0}\right]\right)} \int \sigma_{1}(g) d \operatorname{vol}(g) \times \int e^{4 u} d \operatorname{vol}(g)>0 .
$$

Moreover, as $\nu \rightarrow 0$, the family of solution $\left\{u_{\nu}\right\}$ is bounded in $C^{2}$ norm. Thus, as $\nu \rightarrow 0, u_{\nu}$ converges in $C^{1, \alpha}$ for any $\alpha \in(0,1)$ to some $C^{1,1}$ function $u$. Consequently, this $C^{1,1}$ conformal metric $g_{u}=e^{-2 u} g_{0} \in\left[g_{0}\right] \cap \bar{\Gamma}_{1}^{+}$solves equation (9).

Let us consider the last case $Y_{2,1}\left(\left[g_{0}\right]\right)<0$. As in the previous case, there holds $h(\nu) \leq h(0)=Y_{2,1}\left(\left[g_{0}\right]\right)<0 \forall \nu>0$ if $n \geq 4$, and $h(\nu) \geq$ $h(0)=-Y_{2,1}\left(\left[g_{0}\right]\right)>0 \forall \nu>0$ if $n=3$ and the family of solution $\left\{u_{\nu}\right\}$ is bounded in $C^{2}$ as $\nu \rightarrow 0$. We claim this family is also bounded in $C^{2, \alpha}$ for $\alpha>0$. For this purpose, we will write equation (82) in the new form. Recall $w_{i j}=\nabla_{i j}^{2} u+u_{i} u_{j}-\frac{|\nabla u|^{2}}{2}\left(g_{0}\right)_{i j}+\left(S_{g_{0}}\right)_{i j}$. We define a new second order tensor $\tilde{W}=\left(\tilde{w}_{i j}\right)$ with

$$
\tilde{w}_{i j}=w_{i j}+\mu\left(g_{0}\right)_{i j}
$$


where $\mu$ is some small positive constant to be fixed later. It is clear that

$$
\sigma_{1}(\tilde{W})=\sigma_{1}(W)+n \mu
$$

and

$$
2 \sigma_{2}(\tilde{W})=2 \sigma_{2}(W)+2(n-1) \mu \sigma_{1}(W)+n(n-1) \mu^{2} .
$$

Denote $u_{\nu}$ the unique solution of (82) with $\int \sigma_{1}(g) d v o l(g)=1$ for $k=h(\nu)$ and for all small $\nu>0$. Using (97) and (98), $u_{\nu}$ satisfies the following equation

$$
\frac{\sigma_{2}(\tilde{W})-\frac{n(n-1) \mu^{2}}{2}-(n-1) \mu\left(\sigma_{1}(\tilde{W})-n \mu\right)-\nu}{\sigma_{1}(\tilde{W})-n \mu}=h(\nu) e^{-2 u_{\nu}},
$$

that is, $u_{\nu}$ solves

$$
\frac{\sigma_{2}(\tilde{W})}{\sigma_{1}(\tilde{W})}+\frac{-\nu+n \mu h(\nu) e^{-2 u_{\nu}}+\frac{n(n-1) \mu^{2}}{2}}{\sigma_{1}(\tilde{W})}=h(\nu) e^{-2 u_{\nu}}+(n-1) \mu
$$

Choose a small $\mu>0$ such that $\forall \nu \in\left(0, \nu_{0}\right)$ and $\forall x \in M$, we have

$$
h(\nu) e^{-2 u_{\nu}(x)}+(n-1) \mu<0 .
$$

Now equation (100) is uniformly elliptic and concave for all $\nu \in\left(0, \nu_{0}\right)$. From the classical Krylov's result, the set of solutions $\left\{u_{\nu}\right\}$ is also bounded in $C^{2, \alpha}$ for $\alpha>0$. Passing to the limit, $u_{\nu}$ converges in $C^{2, \alpha^{\prime}}$ for any $\alpha^{\prime} \in(0, \alpha)$ to some $C^{2, \alpha}$ function $u \in \bar{\Gamma}_{1}^{+}$, which is a solution of (10). Finally, writing equation in the form (100) for $\nu=0$, the uniqueness comes from the maximum principle as in the proof of Proposition 1. Therefore, we finish the proof.

q.e.d.

Proof of Theorem 1 . When $\lambda\left(g_{0}, \sigma_{2}\right)>0$, the result follows from Proposition 3. When $\lambda\left(g_{0}, \sigma_{2}\right)=0$, the proof is the same as in the case $Y_{2,1}\left(\left[g_{0}\right]\right)=0$ in the previous proof. $\quad$ q.e.d.

Proof of Corollary 1. It follows from Proposition 3 directly. $\quad$ q.e.d.

Proof of Corollary 3. From Theorem 2, the cone $\Gamma_{2}^{+}$is not empty. Set $g \in \mathcal{C}_{2}\left(\left[g_{0}\right]\right)$. From a result due to Gursky-Viaclovsky $[\mathbf{3 0}]$ the Ricci tensor of $g$ is pointwise positive. Thanks to the result of Hamilton [35] by using the Ricci flow, $M$ is diffeomorphic to a compact 3-dimensional Riemannian manifold with constant curvature. Moreover, $M$ is diffeomorphic to $\mathbb{S}^{3} / \Gamma$, where $\Gamma$ is a finite isometry subgroup of $\mathbb{S}^{3}$ in the standard metric.

q.e.d.

Remark 5. In Corollary 2, the condition $\int_{M^{3}} \sigma_{2}\left(g_{0}\right) d v o l\left(g_{0}\right)>0$ can be easily weakened to $\int_{M^{3}} \sigma_{2}\left(g_{0}\right) \operatorname{dvol}\left(g_{0}\right) \geq 0$. In this case, we have two cases: either (i) there is a conformal metric $g \in \mathcal{C}^{1}\left(\left[g_{0}\right]\right)$ with $\int_{M^{3}} \sigma_{2}(g) \operatorname{dvol}(g)>0$, or (ii) there is no conformal metric $g \in$ 
$\mathcal{C}^{1}\left(\left[g_{0}\right]\right)$ with $\int_{M^{3}} \sigma_{2}(g) d \operatorname{vol}(g) 0$. Case (i) is just Corollary 1. In case (ii), $Y_{2,1}\left(\left[g_{0}\right]\right)=0$ and $g_{0}$ achieves $Y_{2,1}\left(\left[g_{0}\right]\right)$, hence $\sigma_{2}\left(g_{0}\right)=0$. This also implies that $R i c_{g_{0}} \geq 0$ and $M^{3}$ is a quotient of a sphere, since $Y_{1}\left(\left[g_{0}\right]\right)>0$.

\section{The Yamabe invariants $Y_{2,1}\left(\left[g_{0}\right]\right)$}

In this section, we prove Theorem 3. To this aim, we need a technic result.

Lemma 5. Let $\left(M^{n}, g_{0}\right)$ be a compact Riemannian manifold with $g_{0} \in \Gamma_{1}^{+}$and $n \geq 3$. At most one of the followings holds:

1) $M$ admits a regular metric $g \in\left[g_{0}\right] \cap \Gamma_{2}^{+}$;

2) equation (9) admits a $C^{1,1}$ solution $g \in\left[g_{0}\right] \cap \bar{\Gamma}_{1}^{+}$;

3) equation (10) admits a regular solution $g \in\left[g_{0}\right] \cap \bar{\Gamma}_{1}^{+}$.

Proof. Let $g \in\left[g_{0}\right] \cap \Gamma_{2}^{+}$. Without loss of generality, assume $g=g_{0}$. From a result of Guan-Wang [28], there is no $C^{1,1}$ solution of equation (9) in $\bar{\Gamma}_{1}^{+}$. On the other hand, for any $g=e^{-2 u} g_{0} \in \mathcal{C}_{1}\left(\left[g_{0}\right]\right)$, let $x_{0}$ be a minimum point of $u$. At this point, we get $\nabla u=0$ and the Hessian matrix $\nabla^{2} u$ is non-negative definite. Thus, the Schouten tensor at point $x_{0}$ is in $\Gamma_{2}^{+}$. As a consequence, equation (10) does not admit a regular solution $g \in\left[g_{0}\right] \cap \bar{\Gamma}_{1}^{+}$.

Now suppose $g_{1}=e^{-2 u_{1}} g_{0}$ is a regular solution of equation (10) in $\left[g_{0}\right] \cap \bar{\Gamma}_{1}^{+}$. As above, $\left[g_{0}\right] \cap \Gamma_{2}^{+}=\emptyset$. As in the proof of Theorem $2, u_{1}$ solves

$$
\frac{\sigma_{2}(\tilde{W})}{\sigma_{1}(\tilde{W})}+\frac{-n \mu e^{-2 u_{1}}+\frac{n(n-1) \mu^{2}}{2}}{\sigma_{1}(\tilde{W})}=-e^{-2 u_{1}}+(n-1) \mu
$$

where $\mu>0$ is a small positive number such that $f(x):=-e^{-2 u_{1}}+$ $\frac{(n-1) \mu}{2}<0$ and $\tilde{w}_{i j}=w_{i j}+\mu\left(g_{0}\right)_{i j}$. Let $g_{2}=e^{-2 u_{2}} g_{0} \in\left[g_{0}\right] \cap \bar{\Gamma}_{1}^{+}$be a $C^{1,1}$ solution of equation (9). Thus, $u_{2}$ is a subsolution to (102), that is,

$$
\frac{\sigma_{2}(\tilde{W})}{\sigma_{1}(\tilde{W})}+\frac{n \mu f(x)}{\sigma_{1}(\tilde{W})} \geq-e^{-2 u_{1}}+(n-1) \mu,
$$

since $u_{2}$ solves

$$
\frac{\sigma_{2}(\tilde{W})}{\sigma_{1}(\tilde{W})}+\frac{n(n-1) \mu^{2}}{2 \sigma_{1}(\tilde{W})}=(n-1) \mu
$$

and

$$
\sigma_{1}(\tilde{W}) \geq n \mu
$$


Set $v=u_{2}-u_{1}$ and $H(W, x)=\frac{\sigma_{2}(W)+n \mu f(x)}{\sigma_{1}(W)}$. Denote

$$
H^{i j}:=\frac{\partial H(W, x)}{\partial w_{i j}} .
$$

On the other hand, for any $c \in \mathbb{R}, u_{2}+c$ is also a $C^{1,1}$ solution of equation (9). Without loss of generality, we could suppose $v \leq 0$ and $\max v=0$. From (102) and (103), $v$ is a subsolution of some uniformly elliptic second order operator, that is,

$$
L v:=\sum_{i, j} A^{i j} \nabla_{i j}^{2} v+\sum_{i} B^{i} \nabla_{i} v \geq 0
$$

where

$$
A^{i j}=\int_{0}^{1} H^{i j}\left(s \tilde{W}_{2}+(1-s) \tilde{W}_{1}\right) d s .
$$

From the strong maximum principle, $v \equiv 0$. This contradiction yields the desired result and we finish the proof of Lemma. q.e.d.

Proof of Theorem 3. It follows from Theorem 2 and Lemma 5. q.e.d.

By Theorem 2 and Lemma 5, we have

Proposition 4. Let $\left(M^{n}, g_{0}\right)$ be a compact Riemannian manifold with $g_{0} \in \Gamma_{1}^{+}$and $n \geq 3$. The following holds

1) If there is a regular conformal metric $g \in\left[g_{0}\right] \cap \Gamma_{2}^{+}$, then $Y_{2,1}\left(\left[g_{0}\right]\right)>0$;

2) If there is a $C^{1,1}$ conformal metric $g \in\left[g_{0}\right] \cap \bar{\Gamma}_{1}^{+}$satisfying equation (9), then $Y_{2,1}\left(\left[g_{0}\right]\right)=0$;

3) If there is a regular conformal metric $g \in\left[g_{0}\right] \cap \bar{\Gamma}_{1}^{+}$solving equation (10), then $Y_{2,1}\left(\left[g_{0}\right]\right)<0$.

Remark 6. With the same arguments, we know : let $\left(M^{n}, g_{0}\right)$ be a compact Riemannian manifold with $g_{0} \in \Gamma_{1}^{+}$and $n \geq 3$. At most one of the followings holds:

1) $M$ admits a regular metric $g \in\left[g_{0}\right] \cap \bar{\Gamma}_{2}^{+}$;

2) equation (10) admits a regular solution $g \in\left[g_{0}\right] \cap \bar{\Gamma}_{1}^{+}$.

To this aim, let $g_{2}=e^{-2 u_{2}} g_{0} \in\left[g_{0}\right] \cap \bar{\Gamma}_{2}^{+}$. Thus, $u_{2}$ is also a subsolution to (102) and the desired result yields. A direct consequence is that $Y_{2,1}\left(\left[g_{0}\right]\right) \geq 0$ provided $\left[g_{0}\right] \cap \bar{\Gamma}_{2}^{+} \neq \emptyset$.

Finally, we state the following result.

Corollary 7. Let $\left(M, g_{0}\right)$ be a compact Riemannian manifold with positive scalar curvature and of dimension $n \geq 4$. The sign of $\lambda\left(g_{0}, \sigma_{2}\right)$, equivalently the sign of $Y_{2,1}\left(\left[g_{0}\right]\right)$, is a conformal invariant. 


\section{Geometric Applications}

First we show that any manifold in class $\left(1_{+}\right)$admits a psc metric with negative Yamabe constant $Y_{2,1}<0$. Therefore by Theorem 2 we can deform this metric in its conformal class to a metric with non-negative scalar curvature and negative $\sigma_{2}$-scalar curvature. Then we show that $Y_{2,1}$ is continuous in a suitable sense.

Proof of Theorem 4. With the analysis established above, in order to show the Theorem, We need only to show that for any manifold in $\left(1_{+}\right)$ there is a psc metric $g$ with $\int \sigma_{2}(g) \operatorname{dvol}(g)<0$.

Here we use the well-known construction of Gromov-Lawson [20] for positive scalar curvature metrics. See also [51]. Let $S^{p}$ be an embedded sphere in $M$ with trivial normal bundle of codimension $q=n-p \geq 3$. Let $\mathbb{S}^{p} \times D^{q}(\bar{r})$ be an embedding into $M$ for some small constant $\bar{r}>0$. Let $r_{0}$ be a constant fixed later as small as we want.

By the construction of Gromov-Lawson, we have a manifold $\left(N_{1}, h_{1}\right)$ with an end $\mathbb{S}^{p} \times \mathbb{S}^{q-1} \times[0,+\infty)$ such that $N_{1}-\left(\mathbb{S}^{p} \times \mathbb{S}^{q-1} \times[1, \infty], h_{1}\right)$ is isometric to $M-\mathbb{S}^{p} \times D^{q}(\bar{r})$ and $\left(\mathbb{S}^{p} \times \mathbb{S}^{q-1} \times[2,+\infty), h_{1}\right)$ is isometric to $\mathbb{S}^{p} \times \mathbb{S}^{q-1}\left(r_{\infty}\right) \times[2,+\infty)$ with the product metric. Here $\mathbb{S}^{p}$ is standard sphere with radius 1 and $\mathbb{S}^{q-1}\left(r_{\infty}\right)$ is the standard sphere with a small radius $r_{\infty}$. The crucial point in the construction of Gromov-Lawson is that the scalar curvature of $h_{1}$ is positive. Now we glue on $N_{1}-$ $\mathbb{S}^{p} \times \mathbb{S}^{q-1}\left(r_{\infty}\right) \times\left[\tau_{0},+\infty\right)$ a product manifold $\mathbb{S}^{p} \times D^{q}$, where $D^{q}$ is not equipped with the flat metric, but a product metric of $\mathbb{S}^{q-1} \times[0, b]$ in a neighborhood of the boundary and of positive scalar curvature on $D^{q}$. The result manifold is of positive scalar curvature and is diffeomorphic to $M$. It contains a product $\mathbb{S}^{p} \times \mathbb{S}^{q-1}\left(r_{\infty}\right) \times\left[2, \tau_{0}\right]$ for a small $r_{\infty}$ and a large $\tau_{0}$.

Now we consider the case $q=3$. In this case, its Ricci curvature, written as a diagonal matrix, is $\operatorname{diag}\left\{n-4, \cdots, n-4, r_{\infty}^{-2}, r_{\infty}^{-2}, 0\right\}$. Its Schouten tensor, also written as a diagonal matrix and up to a multiple constant independent of $r_{\infty}$, is

$$
\operatorname{diag}\left\{n-4-\alpha, \cdots, n-4-\alpha, r_{\infty}^{-2}-\alpha, r_{\infty}^{-2}-\alpha,-\alpha\right\}
$$

with

$$
\alpha=\frac{1}{2(n-1)}\left((n-3)(n-4)+\frac{2}{r_{\infty}^{2}}\right) .
$$

It can be written as

$$
\frac{1}{n-1} r_{\infty}^{-2} \operatorname{diag}\{-1, \cdots,-1, n-2, n-2,-1\}+C,
$$

Where $C$ is a matrix whose entries are independent of $r_{\infty}$. To decide the sign of $\sigma_{2}$-scalar curvature for this product, we only need to compute $\sigma_{2}(-1, \cdots,-1, n-2, n-2,-1)=-\frac{1}{2}(n-1)(n-2)$. Hence choosing 
$r_{\infty}$ small, we have negative $\sigma_{2}$-scalar curvature in this product part. By choosing $\tau_{0}$ large enough, we obtain a metric $\tilde{g}$ on $M$ with

$$
\int_{M} \sigma_{2}(\tilde{g})<0
$$

Therefore for the conformal class $[\tilde{g}]$ we have $Y_{2,1}([\tilde{g}])<0$. By Theorem 2 , we have a conformal metric $\tilde{h}$ with $\sigma_{2}(\tilde{h}) \leq 0$ and with non-negative scalar curvature.

q.e.d.

When $n=4$, we have another proof. In this case,

$$
\int\left(\sigma_{2}(g)+\frac{1}{16}|W|^{2}\right) d v o l(g)
$$

is the Euler characteristic of $M$ up to a constant multiple. Since $\int|W|^{2} d \operatorname{vol}(g)$ is invariant in a conformal class, so is $\int \sigma_{2}(g) d v o l(g)$. In $[\mathbf{1}]$ the authors constructed a sequence of metrics $g_{i}$ satisfying that

$$
Y_{1}\left(\left[g_{i}\right]\right) \rightarrow Y_{1}\left(\left[g_{0}\right]\right) \text { and } \int|W|^{2} \operatorname{dvol}\left(g_{i}\right) \rightarrow \infty,
$$

as $i \rightarrow \infty$. Since $R_{g_{0}}>0$, from this sequence we can find a psc metric $g_{i}$ with $\int \sigma_{2}\left(g_{i}\right) d v o l\left(g_{i}\right)<0$.

Lemma 6. Let $n \geq 4$. If the space of psc metric is equipped with the $C^{4, \alpha}$-topology, then map $g \rightarrow Y_{2,1}([g])$ is continuous.

Proof. A similar Lemma for the first Yamabe constant $Y_{1}$ was given in [4], see also [5]. For $Y_{2,1}$, the proof becomes complicated. We use solutions of equations considered here.

Let $g_{j}$ be a sequence of psc metric converging to $g$ in $C^{4, \alpha}$ topology. The proof is trivial for the case $n=4$, since $\int_{M} \sigma_{2}(g) d v o l(g)$ is constant in a conformal class. Now we consider the cases $n \geq 5$. By taking a subseqeunce we may assume that $\lim _{j \rightarrow \infty} Y_{2,1}\left(\left[g_{j}\right]\right)=Y_{0}$. It is easy to see that

$$
Y_{0}=\lim _{j \rightarrow \infty} Y_{2,1}\left(\left[g_{j}\right]\right) \leq Y_{2,1}([g]) .
$$

To see this, for any $\varepsilon>0$ find $g_{u}=e^{-2 u} g \in \mathcal{C}_{1}([g])$ such that

$$
\int \sigma_{2}\left(g_{u}\right) \operatorname{dvol}\left(g_{u}\right)<Y_{2,1}([g])+\frac{1}{2} \varepsilon \quad \text { and } \quad \int \sigma_{1}\left(g_{u}\right) d \operatorname{vol}\left(g_{u}\right)=1 .
$$

For sufficiently large $j$, we have $e^{-2 u} g_{i} \in \mathcal{C}_{1}\left(\left[g_{j}\right]\right)$. Since $\int \sigma_{2}$ and $\int \sigma_{1}$ are continuous for this fixed function $u$, we have

$$
Y_{2,1}\left(\left[g_{j}\right]\right) \leq Y_{2,1}([g])+\varepsilon,
$$

for sufficiently large $j$, and hence (106).

Now we show that $Y_{0} \geq Y_{2,1}([g])$. Let $Y_{2,1}\left(\mathbb{S}^{n}\right)$ be the second Yamabe constant for the standard sphere $\mathbb{S}^{n}$. Using a method given in [54], one can show that

$$
Y_{2,1}\left(\mathbb{S}^{n}\right) \geq Y_{2,1}([g]),
$$


for any psc metric $g$. Without loss of generality, we may assume $Y_{0}<$ $Y_{2,1}\left(\mathbb{S}^{n}\right)$. Otherwise, we have $\lim _{j \rightarrow \infty} Y_{2,1}\left(\left[g_{j}\right]\right)=Y_{0}=Y_{2,1}\left(\mathbb{S}^{n}\right) \geq$ $Y_{2,1}([g])$ and hence we are done.

Consider the following perturbed energy functional

$$
J_{\varepsilon, \nu}(g):=\frac{\left.\int\left(\sigma_{2}(g)-\nu e^{4 u}\right) d v o l(g)\right)}{\left(\int e^{2 \varepsilon u} \sigma_{1}(g) d v o l(g)\right)^{\frac{n-4}{n-2-2 \varepsilon}}}
$$

for small $\nu \geq 0$ and $\varepsilon \geq 0$ in the cone $\mathcal{C}_{1}$. The analysis established above implies that the above functional admits a minimizer in $\mathcal{C}_{1}([g])$ for any psc metric. It is easy to check that for given $j \in \mathbb{N}$, there are small constants $\varepsilon_{j} \geq 0$ and $\nu_{j} \geq 0$ such that a minimizer $\tilde{g}_{j}=e^{-2 u_{j}} g_{j} \in$ $\mathcal{C}_{1}\left(\left[g_{j}\right]\right)$ of the perturbed functional $J_{\varepsilon_{j}, \nu_{j}}$ satisfies

$$
J_{\varepsilon_{j}, \nu_{j}}\left(\tilde{g}_{j}\right) \leq Y_{2,1}\left(\left[g_{j}\right]\right)+\frac{1}{j}
$$

(When $Y_{0} \leq 0$, we can take $\varepsilon_{j} \equiv 0$. When $Y_{0}>0$, we can take $\nu_{j} \equiv 0$.) As a minimizer of $J_{\varepsilon, \nu}, \tilde{g}_{j}=e^{-2 u_{j}} g_{j}$ satisfies an equation similar to (8), for which we have local estimates. Since $g_{j}$ converges to $g$ in $C^{4, \alpha}$, the local estimates and $Y_{0}<Y_{2,1}\left(\mathbb{S}^{n}\right)$ imply that $\tilde{g}_{j}$ converges to a metric $\tilde{g}=e^{-2 \tilde{u}} g \in \overline{\mathcal{C}}_{1}([g])$ in $C^{0}$-topology, and hence in $C^{1, \beta}$-topology for any $\beta \in(0,1)$. We first show that

$$
\lim _{j \rightarrow \infty} \int \sigma_{2}\left(e^{-2 u_{j}} g_{j}\right) d \operatorname{vol}\left(e^{-2 u_{j}} g_{j}\right)=\lim _{j \rightarrow \infty} \int \sigma_{2}\left(e^{-2 u_{j}} g\right) d v o l\left(e^{-2 u_{j}} g\right)
$$

and

$\lim _{j \rightarrow \infty} \int e^{2 \varepsilon_{j} u_{j}} \sigma_{1}\left(e^{-2 u_{j}} g_{j}\right) d v o l\left(e^{-2 u_{j}} g_{j}\right)=\lim _{j \rightarrow \infty} \int \sigma_{1}\left(e^{-2 u_{j}} g\right) d v o l\left(e^{-2 u_{j}} g\right)$.

This is clear, for $u_{j}$ has a uniform $C^{2}$ bound and $g_{j}$ converges to $g$ in $C^{4, \alpha}$-topology and $\varepsilon_{j} \rightarrow 0$. Then, we claim that there exists metrics $e^{-2 \tilde{u}_{j}} g \in \mathcal{C}_{1}([g])$ such that

$$
\lim _{j \rightarrow \infty} \int \sigma_{2}\left(e^{-2 \tilde{u}_{j}} g\right) d v o l\left(e^{-2 \tilde{u}_{j}} g\right)=\lim _{j \rightarrow \infty} \int \sigma_{2}\left(e^{-2 u_{j}} g\right) d v o l\left(e^{-2 u_{j}} g\right)
$$

and

$$
\lim _{j \rightarrow \infty} \int \sigma_{1}\left(e^{-2 \tilde{u}_{j}} g\right) d v o l\left(e^{-2 \tilde{u}_{j}} g\right)=\lim _{j \rightarrow \infty} \int \sigma_{1}\left(e^{-2 u_{j}} g\right) d v o l\left(e^{-2 u_{j}} g\right) .
$$


Given $j \in \mathbb{N}$, we choose $t_{j} \in(0,1)$ with $\lim t_{j}=1$ such that $e^{-2 t_{j} u_{j}} g \in$ $\mathcal{C}_{1}([g])$. To see this, we compute

$$
\begin{aligned}
& e^{-2 t_{j} u_{j}} \sigma_{1}\left(e^{-2 t_{j} u_{j}} g\right) \\
= & t_{j} \Delta_{g} u_{j}-t_{j}^{2} \frac{n-2}{2}\left|\nabla u_{j}\right|_{g}^{2}+\sigma_{1}(g) \\
= & t_{j} e^{-2 u_{j}} \sigma_{1}\left(e^{-2 u_{j}} g_{j}\right)+t_{j}\left(\Delta_{g} u_{j}-\Delta_{g_{j}} u_{j}+\sigma_{1}(g)-\sigma_{1}\left(g_{j}\right)\right) \\
& +t_{j}\left(\frac{n-2}{2}\left|\nabla u_{j}\right|_{g_{j}}^{2}-\frac{n-2}{2}\left|\nabla u_{j}\right|_{g}^{2}\right) \\
& +\left(1-t_{j}\right)\left(t_{j} \frac{n-2}{2}\left|\nabla u_{j}\right|_{g}^{2}+\sigma_{1}(g)\right) .
\end{aligned}
$$

It is clear that

$$
\begin{aligned}
& \left|t_{j}\left(\Delta_{g} u_{j}-\Delta_{g_{j}} u_{j}+\sigma_{1}(g)-\sigma_{1}\left(g_{j}\right)\right)+t_{j}\left(\frac{n-2}{2}\left|\nabla u_{j}\right|_{g_{j}}^{2}-\frac{n-2}{2}\left|\nabla u_{j}\right|_{g}^{2}\right)\right| \\
& \quad \leq C\left\|g-g_{j}\right\|_{C^{2}}\left(\left|\nabla u_{j}\right|_{g}^{2}+1\right) .
\end{aligned}
$$

Thus, we can choose $t_{j}$ close to 1 such that $e^{-2 t_{j} u_{j}} g \in \mathcal{C}_{1}([g])$. From (20), (23) and the fact that $u_{j}$ has a uniform $C^{2}$ bound, we have (109) and (110), which imply that $Y_{0}=\lim _{j \rightarrow \infty} Y_{2,1}\left(\left[g_{j}\right]\right) \geq Y_{2,1}([g])$. Hence we finish the proof of the Lemma.

q.e.d.

In fact, the Lemma is true for $C^{4}$-topology.

Proof of Theorem 5. If $M$ belongs to class $\left(2_{+}\right)$, then there is a psc metric of positive $\sigma_{2}$-scalar curvature. By Theorem $3, Y_{2,1}([g])>0$. From Theorem 4 we have another psc metric $\tilde{g}$ with $Y_{2,1}([\tilde{g}])<0$. Using the previous Lemma we have the third psc metric $\hat{g}$ with $Y_{2,1}([\hat{g}])=0$. Now 1. follows from Theorem 2. The proof for 2. and 3 . is the same.

q.e.d.

In order to prove the Conjecture (Trichotomy Theorem) as in $[\mathbf{4 1}]$ and $[42]$, we need an implicit function theorem for the linearization operator of $\sigma_{2}(g)$ in $L^{2, p}$. This seems to be rather difficult for us, at least at the moment.

Example 1. Let us consider the manifold $M=\mathbb{S}^{3} \times \mathbb{S}^{1}$. It is a locally conformally flat manifold with $\sigma_{1}>0$ and $\sigma_{2}=0$ for the product metric. From

$$
2 \pi^{2} \chi(M)=\int_{M} \sigma_{2}(g)+\frac{1}{16}|W|^{2},
$$

we have $\int_{\mathbb{S}^{3} \times \mathbb{S}^{1}} \sigma_{2}(g)=-\frac{1}{16} \int|W|^{2} \leq 0$ for any metric $g$ on $\mathbb{S}^{3} \times \mathbb{S}^{1}$. Hence, $\mathbb{S}^{3} \times \mathbb{S}^{1}$ belongs to class $\left(2_{0}\right)$. 
Example 2. For $n>4$ let us consider the locally conformally flat manifold $\mathbb{S}^{p} \times H^{n-p}$, where $p>n / 2$ and $H^{n-p}$ is a compact quotient of the hyperbolic space $\mathbb{H}^{n-p}$ with sectional curvature -1 . Let $g_{0}$ be the product metric. It is clear that $\sigma_{1}\left(g_{0}\right)>0$, for $p>n / 2$. Its $\sigma_{2}$-scalar curvature, up to a positive constant multiple, is

$$
\left(p-\frac{n}{2}\right)^{2}-\frac{n}{4}
$$

Hence, $p>\frac{n+\sqrt{n}}{2}$ if and only if $\sigma_{2}\left(g_{0}\right)>0$. Now we consider such $p$ with $\sigma_{2}\left(g_{0}\right)=0$, namely $p=\frac{n+\sqrt{n}}{2}$. It is clear that we have to consider $n=(2 m+1)^{2}$ for some $m \in \mathbb{N}$. For example $m=1, M=\mathbb{S}^{6} \times H^{3}$. In general $M_{m}:=\mathbb{S}^{(m+1)(2 m+1)} \times H^{m(2 m+1)}$. We conjecture that on such a manifold there is a psc metric with positive $\sigma_{2}$-scalar curvature. A rough idea to check this can be made as follows. By Theorem 2, if it is not true, we know that any psc metric $g$ on $M_{m}$ has $Y_{2,1}([g]) \leq 0$, which is equivalent to

$$
\inf _{\tilde{g} \in \mathcal{C}_{1}([g])} \int_{M} \sigma_{2}(\tilde{g}) \operatorname{dvol}(\tilde{g}) \leq 0 .
$$

Hence 0 is be a minimax value of $\int_{M} \sigma_{2}(g) d v o l(g)$ and the product metric $g_{0}$ would be a critical point of $\int \sigma_{2}$ on the space of all metrics. If this is true, then a result in $[\mathbf{4 0}]$ implies that $g_{0}$ is a metric of constant sectional curvature. This certainly is false. From this example, we propose a

Conjecture. When $n>4$, class $\left(2_{0}\right)$ is empty.

\section{Further applications in fully nonlinear equations}

With the method considered in this paper, we can also deal with the following problems.

1. When $\left(M, g_{0}\right)$ is a locally conformally flat manifold with $g_{0} \in \mathcal{C}_{k-1}$ $(k>1)$, the method presented here can be used to study the equation

$$
\frac{\sigma_{k}(g)}{\sigma_{k-1}(g)}=f
$$

Similarly we define a Yamabe type invariant in $[\mathbf{2 2}]$ as follows

$$
Y_{k, k-1}\left(\left[g_{0}\right]\right)=\inf \frac{\int_{M} \sigma_{k}(g) d v o l(g)}{\left(\int_{M} \sigma_{k-1}(g) d v o l(g)\right)^{\frac{n-2 k}{n-2 k+2}}},
$$

for $k \leq n / 2$. We can show that if $Y_{k, k-1}>0\left(Y_{k, k-1}=0\right.$ and $Y_{k, k-1}<0$ resp.), then there is a conformal metric $g$ with $\sigma_{k}>0\left(\sigma_{k}=0\right.$ and $\sigma_{k} \leq 0$ resp.) 
2. Let $\left(M^{4}, g_{0}\right)$ be a compact 4-dimensional manifold with positive scalar curvature. One can consider the equation

$$
\frac{\sigma_{2}-s|W|^{2}}{\sigma_{1}}=f
$$

where $W$ is the Weyl tensor and $s$ is a non-negative number. In this case for a fixed $s$

$$
\int\left(\sigma_{2}-s|W|^{2}\right) d \operatorname{vol}(g)
$$

is a constant in a given conformal class. With the method presented here, we can show that the number $\int\left(\sigma_{2}-s|W|^{2}\right) \operatorname{dvol}(g)$ is positive, null or negative resp. if and only if there is a conformal metric with

$$
\sigma_{2}-s|W|^{2}>0,=0 \text { or } \leq 0 \text { resp. }
$$

The positive and null cases were studied already in $[\mathbf{1 2}]$ as mentioned above. This can be seen as a generalization of the following classical result: Let $\left(M^{2}, g_{0}\right)$ be a closed surface. Its Euler characteristic $\chi\left(M^{2}\right)=\frac{1}{2 \pi} \int_{M} R_{g} d v o l(g)$ is positive, negative or null resp. if and only if there is a conformal metric $g$ with positive, negative or null scalar curvature resp. It is clear that equation (114) can also be considered on a higher dimensional manifold.

3. Our methods can also be applied to study the following fully nonlinear equations

$$
\frac{\sigma_{k}\left(\nabla^{2} u\right)}{\sigma_{k-1}\left(\nabla^{2} u\right)}=f
$$

and

$$
\frac{\sigma_{k}\left(\nabla^{2} u+u g\right)}{\sigma_{k-1}\left(\nabla^{2} u+u g\right)}=f,
$$

in the class of $(k-1)$-admissible functions.

4. Another interesting problem is a generalization of the prescribed scalar curvature problem. Let $M^{n}=\mathbb{S}^{n}$ and $f: \mathbb{S}^{n} \rightarrow \mathbb{R}^{1}$ be a smooth function. We would like to ask if there is a conformal metric $g \in \overline{\mathcal{C}}_{1}$ such that

$$
\sigma_{2}(g)=f \sigma_{1}(g) .
$$

A Kazdan-Warner type necessary condition could be obtained as Han [36] did for the prescribed $\sigma_{k}$-scalar curvature problem. Here, and also in the previous problem, the function $f$ need not be positive. 


\section{References}

[1] K. Akutagawa, B. Botvinnik, O. Kobayashi \& H. Seshadri, The Weyl functional near the Yamabe invariant, J. Geom. Anal. 13 (2003) 1-20, MR 1967032, Zbl 1048.53020.

[2] T. Aubin, Métriques riemanniennes et courbure, J. Differential Geom. 4 (1970) 383-424, MR 0279731, Zbl 0212.54102.

[3] T. Aubin, Équations différentilles non linéaires et problème de Yamabe concernant la courbure scalaire, J. Math. Pures Appl. 55 (1976) 269-296, MR 0431287, Zbl 0336.53033.

[4] L. Bérard Bergery, Scalar curvature and isometry groups, in Sepctra of Riemannian manifolds, ed. M. Berger, S. Murakami, T. Ochiai, Kagai, Tokyo, 1983, 9-28.

[5] A. Besse, Einstein manifolds, Ergebnisse der Mathematik und ihrer Grenzgebiete (3) 10 Springer-Verlag, Berlin, 1987, MR 2371700, Zbl 1147.53001.

[6] T. Branson \& A. Gover, Variational status of a class of fully nonlinear curvature prescription problems, Calc. Var. Partial Differential Equations 32 (2008) 253262, MR 2389992, Zbl 1147.53029.

[7] S. Brendle \& J. Viaclovsky, A variational characterization for $\sigma_{n / 2}$, Calc. Var. Partial Differential Equations 20 (2004) 399-402, MR 2071927, Zbl 1059.53033.

[8] L. Caffarelli, L. Nirenberg \& J. Spruck, The Dirichlet problem for nonlinear second-order elliptic equations. III. Functions of the eigenvalues of the Hessian, Acta Math. 155 (1985) 261-301, MR 0806416, Zbl 0654.35031.

[9] G. Catino \& Z. Djadli, Some pinched 3-manifolds are space forms, ArXiv: math.DG/07070338.

[10] A. Chang, M. Gursky \& P. Yang, An equation of Monge-ampère type in conformal geometry, and four manifolds of positive Ricci curvature, Ann. of Math. 155 (2002) 709-787, MR 1923964, Zbl 1031.53062.

[11] A. Chang, M. Gursky \& P. Yang, An a priori estimate for a fully nonlinear equation on Four-manifolds, J. Anal. Math. 87 (2002) 151-186, MR 1945280, Zbl 1067.58028.

[12] A. Chang, M. Gursky \& P. Yang, A conformally invariant sphere theorem in four dimensions, Publ. Math. Inst. Hautes Études Sci. 98 (2003) 105-143, MR 2031200, Zbl 1066.53079.

[13] S. Chen, Local estimates for some fully nonlinear elliptic equations, Int. Math. Res. Not. 2005 no. 55 3403-3425, MR 2204639, Zbl 1159.35343.

[14] S. Dieter, Nonlinear degenerate curvature flows for weakly convex hypersurfaces, Calc. Var. Partial Differential Equations 22 (2005) 229-251, MR 2106768, Zbl 1076.53079.

[15] A. Futaki, Scalar-flat closed manifolds not admitting positive scalar curvature metrics, Invent. Math. 112 (1993) 23-29, MR 1207476, Zbl 0792.53036.

[16] L. Garding, An inequality for hyperbolic polynomials, J. Math. Mech. 8 (1959) 957-965, MR 0113978, Zbl 0090.01603.

[17] Z. Gao \& S.T. Yau, The existence of negatively Ricci curved metrics on threemanifolds, Invent. Math. 85 (1986) 637-652, MR 0848687, Zbl 0603.53025.

[18] Y. Ge \& G. Wang, On a fully nonlinear Yamabe problem, Ann. Sci. École Norm. Sup. 39 (2006) 569-598, MR 2290138, Zbl 1121.53027. 
[19] Y. Ge \& G. Wang, On a quotient conformal equation, Int. Math. Res. Not. 2007 no. 6 rnm 019, MR 2348093, Zbl 1133.53033.

[20] M. Gromov \& H. B. Lawson, The classification of simply connected manifolds of positive scalar curvature, Ann. of Math. (2) 111 (1980) 423-434, MR 0577131, Zbl 0463.53025.

[21] P. Guan, Topics in Geometric Fully Nonlinear Equations, Lecture Notes, http://www.math.mcgill.ca/guan/notes.html

[22] P. Guan, C.-S. Lin \& G. Wang, Application of The Method of Moving Planes to Conformally Invariant Equations, Math. Z. 247 (2004) 1-19, MR 2054518, Zbl 1068.53023.

[23] P. Guan, C.-S. Lin \& G. Wang, Schouten tensor and some topological properties, Comm. Anal. Geom. 13 (2005) 887-902, MR 2216144, Zbl 1110.53025.

[24] P. Guan, C.-S. Lin \& G. Wang, local gradient estimates for conformal quotient equations, Internat. J. Math. 18 (2007) 349-361, MR 2325351, Zbl 1121.53028.

[25] P. Guan, J. Viaclovsky \& G. Wang, Some properties of the Schouten tensor and applications to conformal geometry, Trans. Amer. Math. Soc. 355 (2003) 925-933, MR 1938739, Zbl 1022.53035.

[26] P. Guan \& G. Wang, Local estimates for a class of fully nonlinear equations arising from conformal geometry, Int. Math. Res. Not. 2003 no. 26 1413-1432, MR 1976045, Zbl 1042.53021.

[27] P. Guan \& G. Wang, A fully nonlinear conformal flow on locally conformally flat manifolds, J. Reine Angew. Math. 557 (2003) 219-238, MR 1978409, Zbl 1033.53058 .

[28] P. Guan \& G. Wang, Geometric inequalities on locally conformally flat manifolds, Duke Math. J. 124 (2004) 177-212, MR 2072215, Zbl 1059.53034.

[29] P. Guan \& G. Wang, Conformal deformations of the smallest eigenvalue of the Ricci tensor, Amer. J. Math. 129 (2007) 499-526, MR 2306044, Zbl 1143.53033.

[30] M. Gursky \& J. Viaclovsky, A new variational characterization of threedimensional space forms, Invent. Math. 145 (2001) 251-278, MR 1872547, Zbl 1006.58008.

[31] M. Gursky \& J. Viaclovsky, Volume comparison and the $\sigma_{k}$-Yamabe problem, Advances in Math. 187 (2004) 447-487, MR 2078344, Zbl 1066.53081.

[32] M. Gursky \& J. Viaclovsky, A fully nonlinear equation on 4-manifolds with positive scalar curvature, J. Differential Geom. 63 (2003) 131-154, MR 2015262, Zbl 1070.53018 .

[33] M. Gursky \& J. Viaclovsky, Prescribing symmetric functions of the eigenvalues of the Ricci tensor, Ann. of Math. 166 (2007) 475-531, MR 2373147, Zbl 1142.53027.

[34] M. Gursky \& J. Viaclovsky, Fully nonlinear equations on Riemannian manifolds with negative curvature, Indiana Univ. Math. J. 52 (2003) 399-419, MR 1976082, Zbl 1036.53025.

[35] R. Hamilton, Three-manifolds with positive Ricci curvature, J. Differential Geom. 17 (1982) 255-306, MR 0664497, Zbl 0504.53034.

[36] Z.-C. Han, A Kazdan-Warner type identity for the $\sigma_{k}$ curvature, C. R. Math. Acad. Sci. Paris 342 (2006) 475-478, MR 2214598, Zbl 1099.53028. 
[37] E. Hebey, Nonlinear analysis on manifolds: Sobolev spaces and inequalities. Courant Lecture Notes in Math., 5. Courant Inst. of Math. Sci., New York; Amer. Math. So., Providence, RI, 1999, MR 1688256, Zbl 0981.58006.

[38] N. Hitchin, Harmonic spinors, Advances in Math. 14 (1974) 1-55, MR 0358873, Zbl 0284.58016.

[39] G. Huisken \& C. Sinestrari, Convexity estimates for mean curvature flow and singularities of mean convex surfaces, Acta Math. 183 (1999) 45-70, MR 1719551, Zbl 0992.53051.

[40] Z. Hu \& H. Li, A new variational characterization of $n$-dimensional space forms, Trans. Amer. Math. Soc. 356 (2004) 3005-3023, MR 2052939, Zbl 1058.53029.

[41] J. Kazdan \& F. Warner, Scalar curvature and conformal deformation of Riemannian structure, J. Differential Geom. 10 (1975) 113-134, MR 0365409, Zbl 0296.53037.

[42] J. Kazdan \& F. Warner, Existence and conformal deformation of metrics with prescribed Gaussian and scalar curvatures, Ann. of Math. (2) 101 (1975) 317331, MR 0375153, Zbl 0297.53020.

[43] N. Krylov, Nonlinear elliptic and parabolic equations of the second order, D. Reidel, 1987, MR 0901759, Zbl 0619.35004.

[44] J. Lee \& T. Parker, The Yamabe problem, Bull. Amer. Math. Soc. (N.S.) 17 (1987) 37-91, MR 0888880, Zbl 0633.53062.

[45] A. Li \& Y. Li, On some conformally invariant fully nonlinear equations, Comm. Pure Appl. Math. 56 (2003) 1416-1464, MR 1988895, Zbl 1155.35353.

[46] A. Li \& Y. Li, On some conformally invariant fully nonlinear equations. II. Liouville, Harnack and Yamabe, Acta Math. 195 (2005) 117-154, MR 2233687, Zbl pre05039005.

[47] A. Li \& Y. Li, A Liouville type theorem for some conformally invariant fully nonlinear equations, Geometric analysis of PDE and several complex variables, 321-328, Contemp. Math. 368, Amer. Math. Soc., Providence, RI, 2005, MR 2126479, Zbl 1108.35060.

[48] A. Lichnerowicz, Spineurs harmoniques, C. R. Acad. Sci. Paris 257 (1963) 7-9, MR 0156292, Zbl 0136.18401.

[49] P. L. Lions, Two remarks on the Monge-Ampère, Ann. Mat. Pure Appl. 142 (1985) 263-275, MR 0839040, Zbl 0594.35023.

[50] J. Lohkamp, Metrics of negative Ricci curvature, Ann. of Math. (2) 140 (1994) 655-683, MR 1307899, Zbl 0824.53033.

[51] J. Rosenberg \& S. Stolz, Metrics of positive scalar curvature and connections with surgery, Surveys on surgery theory Vol. 2, 353-386, Ann. of Math. Stud., 149, Princeton Univ. Press, Princeton, NJ, 2001, MR 1818778, Zbl 0971.57003.

[52] R. Schoen, Conformal deformation of a Riemannian metric to constant curvature, J. Differential Geom. 20 (1984) 479-495, MR 0788292, Zbl 0576.53028.

[53] R. Schoen \& S. T. Yau, On the structure of manifolds with positive scalar curvature, Manuscripta Math. 28 (1979) 159-183, MR 0535700, Zbl 0423.53032.

[54] W.M. Sheng, N.Trudinger \& X.-J. Wang, The Yamabe problem for higher order curvatures, J. Differential Geom. 77 (2007) 515-553, MR 2362323, Zbl 1133.53035.

[55] S. Stolz, Simply connected manifolds of positive scalar curvature, Ann. of Math (2) 136 (1992) 511-540, MR 1189863, Zbl 0784.53029. 
[56] S. Stolz, A conjecture concerning positive Ricci curvature and the Witten genus, Math. Ann. 304 (1996) 785-800, MR 1380455, Zbl 0856.53033.

[57] S. Stolz, Manifolds of positive scalar curvature, Topology of high-dimensional manifolds, No. 1, 2 (Trieste, 2001), 661-709, ICTP Lect. Notes, 9, Abdus Salam Int. Cent. Theoret. Phys., Trieste, 2002, MR 1937026, Zbl 1083.53036.

[58] N. Trudinger, On imbeddings into Orlicz spaces and some applications, J. Math. Mech. 17 (1967) 473-483, MR 0216286, Zbl 0163.36402.

[59] N. Trudinger \& X-J. Wang, On Harnack inequalities and singularities of admissible metrics in the Yamabe problem, Calc. Var. Partial Differential Equations 35 (2009) 317-338, MR 2481828, Zbl 1163.53327.

[60] J. Viaclovsky, Conformal geometry, contact geometry and the calculus of variations, Duke Math. J. 101 (2000) 283-316, MR 1738176, Zbl 0990.53035.

[61] J. Viaclovsky, Conformally invariant Monge-Ampère equations: global solutions, Trans. Amer. Math. Soc. 352 (2000) 4371-4379, MR 1694380, Zbl 0951.35044.

[62] J. Viaclovsky, Estimates and some existence results for some fully nonlinear elliptic equations on Riemannian manifolds, Comm. Anal. Geom. 10 (2002) 815-846, MR 1925503, Zbl 1023.58021.

[63] J. Viaclovsky, Conformal geometry and fully nonlinear equations, Inspired by S. S. Chern, 435-460, Nankai Tracts Math. 11 World Sci. Publ., Hackensack, NJ, 2006, MR 2313345, Zbl 1142.53030.

[64] G. Wang, $\sigma_{k}$-scalar curvature and eigenvalues of the Dirac operator, Ann. Global Anal. Geom. 30 (2006) 65-71, MR 2249613, Zbl 1101.53030.

[65] X.-J., Wang, A class of fully nonlinear elliptic equations and related functionals, Indiana Univ. Math. J. 43 (1994) 25-54, MR 1275451, Zbl 0805.35036.

[66] H. Yamabe, On a deformation of Riemannian structures on compact manifolds, Osaka Math. J. 12 (1960) 21-37, MR 0125546, Zbl 0096.37201.

DÉPARTEMEnt De Mathématiques Université Paris Est Créteil VAl de Marne

61 aVenue du GÉnÉral de Gaulle 94010 Créteil Cedex, France

E-mail address: ge@univ-paris12.fr

Department of Mathematics NATIONAL TAIWAN UNIVERSITY AND Taida Institute for Mathematical Science

NATIONAL TAIWAN UNIVERSITY

TAIWAN

E-mail address: cslin@math.ntu.edu.tw

Albert-Ludwigs-Universität Freiburg

MATHEMATISCHES INSTITUT

ECKERSTRA.1

D-79104 Freiburg, Germany

E-mail address: guofang.wang@math.uni-freiburg.de 